\title{
Who is the Master?
}

\author{
Jean-Marc Alliot* \\ IRIT, Toulouse University, CNRS, France
}

\begin{abstract}
There has been debates for years on how to rate chess players living and playing at different periods (see Keene and Divinsky (1989)). Some attempts were made to rank them not on the results of games played, but on the moves played in these games, evaluating these moves with computer programs. However, the previous attempts were subject to different criticisms, regarding the strengths of the programs used, the number of games evaluated, and other methodological problems.

In the current study, 26,000 games (over 2 millions of positions) played at regular time control by all world champions since Wilhelm Steinitz have been analyzed using an extremely strong program running on a cluster of 640 processors. Using this much larger database, the indicators presented in previous studies (along with some new, similar, ones) have been correlated with the outcome of the games. The results of these correlations show that the interpretation of the strength of players based on the similarity of their moves with the ones played by the computer is not as straightforward as it might seem.

Then, to overcome these difficulties, a new Markovian interpretation of the game of chess is proposed, which enables to create, using the same database, Markovian matrices for each year a player was active. By using classical linear algebra methods on these matrices, the outcome of games between any players can be predicted, and this prediction is shown to be at least as good as the classical ELO prediction for players who actually played against each others.
\end{abstract}

Keywords: Chess, Player Evaluation

\section{INTRODUCTION}

The ranking of players in general, and especially of chess players, has been studied for almost 80 years. There were many different systems until 1970 such as the Ingo system (1948) designed by Anton Hoesslinger and used by the German federation, the Harkness system (1956) designed by Kenneth Harkness (1967) and used by the USCF federation, and the English system designed by Richard Clarke. All these systems, which were mostly "rule of thumb" systems, were replaced in almost every chess federations by the ELO system around 1970. The ELO system, the first to have a sound statistical basis, was designed by Arpad Elo (1978) from the assumption that the performance of a player in a game is a normally distributed random variable. Later on, different systems trying to refine the ELO system were proposed, such as the chessmetrics system designed by Jeff Sonas (2005), or the Glicko system, designed by Mark Glickman (1995), which is used on many online playing sites. All these systems share however a similar goal: to infer a ranking from the results of the games played and not from the moves played (for a comprehensive overview see also Glickman and Jones, 1999).

Guid and Bratko (2006) made a pioneering work, and advocated the idea of ranking players by analyzing with a computer program the moves made and by trying to assert the quality of their moves (see also Guid and Bratko, 2007; 2008; Guid, 2010). However, their work was criticized (Riis, 2006) on different grounds. First, Guid and Bratko used a chess program (CRAFTY) which in 2006 had an ELO rating around 2700, while top chess players have a rating above 2700. Moreover, they used a limited version of CRAFTY which evaluated only 12 plies, which therefore reduces further its playing strength. Second,

\footnotetext{
*Corresponding author: Jean-Marc Alliot, IRIT, Toulouse University, CNRS, France. Tel.: +33 561556765 ; Fax: +33 5 615562 58; E-mail: jean-marc.alliot@irit.fr.
} 
the sample analyzed is small (1397 games with 37,000 positions only). Guid and Bratko (2011) used different and better engines (such as RYBKA 3, with a rating of 3073 ELO at the time). However, the search depth remained low (from 5 to 12), meaning that the real strength of the program was far from 3000 ELO, and the set of games remained small, as they only studied World Chess Championship games. Their results were aggregated (there was no evaluation per year), and not easily reproducible as the database of the evaluations was not put in the public domain. A second problem was that the metrics they used could not be analyzed as the raw results were not available. A similar effort was made by Charles Sullivan (2008). In total 18,875 games were used (which is a much larger sample), but the average ply was only 16, the program used was still CRAFTY, and the raw data were not made available, which makes the discussion of the metrics used (such as "Raw error and Complexity") difficult. This lack of raw data also denies the possibility to try different hypotheses (the author decided for example to evaluate only game turns 8 to 40, which is debatable; Guid and Bratko made the same kind of decisions in their original paper, such as simply excluding results when the score was above or less than 200 centipawns, which is also debatable). All these problems were discussed too by Fatta, Haworth, and Regan (2009) and Haworth, Regan, and Fatta (2010).

In this article I present a database of 26,000 games (the set of all games played at regular time controls by all World Champions from Wilhelm Steinitz to Magnus Carlsen), with more than 2 million positions. All games were analyzed at an average of 2 minutes by move ( 26 plies on the average) by what is currently the best or almost best chess program (STOCKFISH), rated around 3300 ELO at the CCRL rating list. For each position, the database contains the evaluation of the two best moves and of the move actually played, and for each move the evaluation, the depth, the selective depth, the time used, the mean delta between two successive depth and the maximum delta between two successive depths. As the database is in PGN it can be used and analyzed by anyone, and all kind of metrics can be computed from it. The study was performed on the OSIRIM cluster (640 HE 6262 AMD processors) at the Toulouse Computer Science Research Institute, and required 61440 hours of CPU time. The exact methodology is described in section 2.

In section 3 we present different indicators that can be used to evaluate the strength of a player. Some of them were already presented in other papers or other studies such as tactical complexity indicators (section 3.1) in Sullivan (2008), "quality of play"1 (sections 3.2) which was mainly introduced by the seminal work of Guid and Bratko (2006), distribution of gain (section 3.3) introduced by Ferreira (2012). Last, we introduce in section 3.4 a new indicator based on a Markovian interpretation of chess which overcomes some of the problems encountered with the other indicators ${ }^{2}$.

These indicators are then discussed, validated and compared using our database in section 4 . The results found demonstrate that the evaluation of a player's strength based on the "quality" of his moves

\footnotetext{
${ }^{1}$ That we will call in this paper "conformance".

${ }^{2}$ I consider here that computer programs are now strong enough (see next section) to be considered as "nearly perfect" oracles when evaluating human games. This is absolutely true when considering endgames (at least up to 6 pieces): here the evaluation function for each position can return the distance to mate, and thus gives an exact evaluation of each move. Of course, as chess has not been solved, the evaluation function in the middle game is only an approximation of this exact function, and different chess programs might return (a) different best moves ordering, and (b) different evaluation for the same position (STOCKFISH is for example known for returning higher/lower evaluations than its siblings). (b) does not change much to the current work: all results and curves would keep exactly the same shape, only the scales would be modified. (a) is however a more serious objection: would the results be the same if using for example KoмоDO instead of STOCKFISH? The two programs have approximately the same strength and sometimes return different move ordering for the same position. This should be the subject of a further study; there has already been work done on comparing the output of different engines (Levene and Bar-Ilan, 2005), especially recently as a result of the RYBKA controversy (Dailey, Hair, and Watkins, 2013), which shows that programs usually agree on $50 \%$ to $75 \%$ of the moves. However, such articles concentrate mainly on how many moves are different, and not on how much moves are different.
} 
is not as straightforward as it might seem, as there remains a difficult question to answer: who is the best player: the one who finds the exact best move most of the time but can make several mistakes, or the one who does not find the best move as often, but makes smaller mistakes? As shown in the following sections, there is no simple answer to this question; we will see that indicators are difficult to calibrate, that a scalar indicator such as move conformance enables to build a global ranking, but is less accurate than a Markovian predictor which is then more accurate but enables only head to head comparison of players.

\section{METHODOLOGY}

We present in this section the evaluation of the ELO strength of the program (2.1), the criteria used for choosing the games to evaluate (2.2), the experimental settings (2.3), and the kind of information saved in the database (2.4).

\subsection{Evaluation of the ELO strength of the program used}

The choice of STOcKFISH was quite straightforward. STOCKFISH, as of 10/2015, tops the SSDF list (Swedish Chess Computer Association, 2015) and is second on the CCRL list (CCRL website, 2015). It is an open source program, which can be easily compiled and optimized for any linux system. At the SSDF rating list, STOCKFISH is rated 3334 ELO, and 3310 at the CCRL rating list. These ratings are given with the program running with 4 CPUs. STOCKFISH 6 on a single core is only rated at the CCRL list at 3233 ELO. The ratings of the SSDF list are given for a Q6600 processor. STOCKFISH on this processor is computing $3283 \mathrm{kn} / \mathrm{s}$ (kilo-nodes by second) when using 4 cores (Canbaz, 2015). It has however not been benchmarked when using one core but the QX9650 using 4 cores is benchmarked at $4134 \mathrm{kn} / \mathrm{s}$ and at $1099 \mathrm{kn} / \mathrm{s}$ using one core. So it is safe to assess a computation speed of around 870 $\mathrm{kn} / \mathrm{s}$ on a Q6600 using one core.

On a $6262 \mathrm{HE}$ core, STOCKFISH was benchmarked at $630 \mathrm{kn} / \mathrm{s}$, so speed is divided by 1.38 compared to the Q6600. Moreover, the games we are evaluating were played at regular time controls ( $3 \mathrm{~min} / \mathrm{moves}$ on the average) but we only use 2 minutes by move for the evaluation. This induces a second reduction of 1.5 , for a total reduction of almost 2 . There has been different studies on the increase in playing strength regarding the depth of the search and the time used to search (Hyatt, 1997; Heinz, 2001a,b; Ferreira, 2013; Guid and Bratko, 2007 and many others). Considering all these elements, it is safe to assess that such a decrease in speed will not cost more than 80 ELO points, and that STOCKFISH under these test conditions has a rating around 3150 ELO points. This is 300 points higher than the current World Champion Magnus Carlsen at 2840, which is also the highest ELO ever reached by a human player.

The question of whether this 3150 rating, which has only be computed through games with other computer programs, is comparable to the ratings of human players is not easy to answer. Man vs Machine games have become scarcer. There was an annual event in Bilbao called "People vs Computers", but the results in 2005 were extremely favorable to computer programs (Levy, 2005). David Levy, who was the referee of the match, even suggested that games should be played with odds and the event was apparently canceled the next year. In 2005 also, Michael Adams lost 51/2-1/2 to Hydra (a 64 CPU dedicated computer), and in 2006 Vladimir Kramnik, then World Champion, lost 4-2 to DEEP Fritz. In 2009, HiARCs 13 running on a very slow hardware mobile phone (less than $20 \mathrm{kn} / \mathrm{s}$ ) won the Copa Mercosur tournament (a category 6 tournament) in Argentina with 9 wins and 1 draw, and a performance of 2898 ELO (Chessbase, 2009). In the following years there have been matches with odds (often a pawn) which clearly demonstrate the superiority of computer programs, even with odds. 
In 2014, Hiraku Nakamura (2800 ELO) played two games against a "crippled" STOCKFISH (no opening database and no endgame tablebase) with white and pawn odds, lost one game and drew the other. So, even if the 3150 ELO rating of this STOCKFISH 6 test configuration is not $100 \%$ correct, it is pretty safe to assert that it is much stronger than any human player ever.

\subsection{The initial database}

The original idea was to evaluate all games played at regular time controls (40 moves in $2 \mathrm{~h}$ ) by all "World Champions" from Wilhelm Steinitz to Magnus Carlsen. This is of course somewhat arbitrary, as FIDE World Championships only started in 1948, and there was a split from 1993 up to 2006 between FIDE and the Grand Masters Association / Professional Chess Association.

Twenty players were included in the study: Wilhelm Steinitz, Emanuel Lasker, José Raul Capablanca, Alexander Alekhine, Max Euwe, Mikhail Botvinnik, Vasily Smyslov, Mikhail Tal, Tigran Petrosian, Boris Spassky, Robert James Fischer, Anatoly Karpov, Gary Kasparov, Alexander Khalifman, Viswanathan Anand, Ruslan Ponomariov, Rustam Kasimdzhanov, Veselin Topalov, Vladimir Kramnik, and Magnus Carlsen.

Gathering the games was done by using the "usual" sources such as the Chessbase Database, Mark Crowthers' "This Week In Chess" and many other online resources. Scripts and programs were developed to cross-reference all the sources in order to have a final database which was consistent regarding data such as player names or date formatting. In the end, after suppressing duplicates, dubious sources, games with less than 20 game turns, games starting from a non standard position and incorrect games, more than 40,000 games were available.

The second filtering task was to keep only games played at regular time controls. This proved to be a much more difficult task; time controls are usually absent from databases. Some have information regarding "EventType", but it is difficult to make a completely safe job. The option was to suppress all games for which it was almost certain that they were either blitz, rapid, simultaneous or blind games, which eliminated around 15,000 games. However, games played at k.o. time control during the 1998-2004 period were kept; this decision was made in order to keep in the databases the FIDE World Championships which were played at this time control between 1998 and 2004.

The final database consists of 25,802 games with more than 2,000,000 positions. The number of games evaluated for each player is presented in Table 1. The database is probably the weakest point of this study, as it is extremely probable that there are games played at time controls quite different from the standard $2 \mathrm{~h} / 40$ moves. This is not such a problem as long as the difference is not too important, but move quality is certainly inferior in rapid games. However, the goal here is also to provide raw material, and anyone can improve the database by suppressing improperly selected games.

\subsection{The experimental settings}

A meta program was written using MPI (Snir et al., 1995) to dispatch the work on the nodes of the cluster. Each elementary program on each node was communicating with a STOCKFISH 6 instance using the UCI protocol. The Syzygy 6-men tablebase was installed in order to improve endgame play. This revealed a small bug in STOCKFISH 6, and a more recent, github-version, of STOcKFISH, where the bug was corrected, had to be used (version 190915). Hash tables were set to 4GB for each instance. This size was chosen after testing different sizes (2, 4 and 6GB) on a subset of the database. MultiPV was set to 2, for different reasons. First, the best two moves are analyzed in order to have an indicator 
Table 1

Games evaluated for each player

\begin{tabular}{|l|r|r|r|}
\hline Player & White & Black & Total \\
\hline \hline Steinitz & 303 & 302 & 605 \\
Lasker & 301 & 286 & 587 \\
Capablanca & 466 & 375 & 841 \\
Alekhine & 671 & 655 & 1326 \\
Euwe & 729 & 706 & 1435 \\
Botvinnik & 574 & 546 & 1120 \\
Smyslov & 1230 & 1185 & 2415 \\
Tal & 1141 & 1038 & 2179 \\
Petrosian & 970 & 904 & 1874 \\
Spassky & 1044 & 1012 & 2056 \\
Fischer & 374 & 391 & 765 \\
Karpov & 1167 & 987 & 2154 \\
Kasparov & 722 & 718 & 1440 \\
Khalifman & 819 & 749 & 1568 \\
Anand & 888 & 861 & 1769 \\
Ponomariov & 558 & 511 & 1069 \\
Kasimdzhanov & 503 & 510 & 1013 \\
Topalov & 728 & 708 & 1436 \\
Kramnik & 715 & 671 & 1386 \\
Carlsen & 574 & 565 & 1139 \\
\hline
\end{tabular}

of the complexity and of the stability of the position. Second, it is often the case that the move played by the human player is either the first or the second best one. Thus the small percentage of time lost by evaluating 2 lines is at least partly compensated by not having to restart an analysis for the evaluation of the human player's move.

In previous studies, engines were often used at a fixed depth, instead of using them with time controls. Guid (2010) and Guid and Bratko (2011) give two arguments to use fixed depths. On the one hand, fixing the depth gives more time to complex positions, and less to simple positions. This is debatable, as some positions with a high branching factor may be extremely stable in their evaluation, and thus not so complex (this is the case for example at the beginning of a game). On the other hand, they want to avoid the effect of the monotonicity of the evaluation function ${ }^{3}$, which reports larger differences when searching deeper. Thus a position with a computed $\delta=v_{b}-v_{p}$ between the move played and the best move at depth $d$ will probably have a larger $\delta$ when searched at depth $d+1$. So, Guid and Bratko advocate the use of the same depth for all positions in the game, in order to have comparable $\delta$. However, this is debatable also; while the monotonicity of the evaluation function is a fact, it is not clear if this monotonicity evolves faster regarding depth of search ${ }^{4}$, or length of search. The problem of the reproducibility and stability of the evaluation of chess programs has been also discussed in other studies such as the one by Barnes and Castro-Hernandez (2015) regarding cheating in (human) chess by using computers; differences observed are minimal and should not impact this study.

\footnotetext{
${ }^{3}$ In layman's words, the deeper you search, the more important is (usually) the difference in a given position between the best move evaluation and any other move evaluation; it is easy to understand why: when you begin to build a small advantage, you usually improve it as time goes by, which in terms of computer search is just an increase in the depth of the search.

${ }^{4}$ This could however be the start of a more in depth discussion about the structure and the interpretation of the evaluation functions in chess. While in some other games (such as reversi for example as done by Michael Buro for Logistello), the evaluation function returns a probability of winning the game, in chess, it is usually presented as the evaluation of the material on the board, with different correcting terms. However, even if it is built that way, this is not what it is supposed to be. The fact that, in chess, there is no absolute simple mapping between the value of the position and the probability of winning the game is a problem that we will discuss again in subsection 3.2.3. Finding such a mapping is however not a real problem for chess programmers because their problem is more to find a good ranking of the moves in a given position than an evaluation of the probability of winning the game, which has no direct practical interest. See for example (Gomboc, Buro, and Marsland, 2005) for the problem of tuning evaluation functions.
} 
So another solution was adopted. The time limit set for the program on any position was 4 minutes. However, the meta-program which was controlling the engine was permanently monitoring the output, and was analyzing the evolution of the position evaluation during the search. The conditions checked are:

- the engine had searched for at least one minute;

- the two best moves had been evaluated at exactly the same depth (to be sure that the evaluation of the moves are comparable);

- the search had reached an evaluation point and an "info" string containing depth, score and pv (principal variation) had just been returned by the UCI interface.

Then, if these three conditions hold, the search was stopped if:

1. the engine had searched for at least 3 minutes,

2. or the position analyzed was strongly biased in favor of the same player in successive game turns,

3. or the search was stable (the differences between evaluations for two successive depths was small) for successive depths.

Condition 2 stops the search if the position is steadily biased in the same direction for at least three consecutive game turns ${ }^{5}\left(e_{0} \times e_{1}<0\right.$ and $\left.e_{1} \times e_{2}<0\right)$ in the game and if the time already used (in minutes) is greater than:

$$
4 \times \max \left(100,\left(1000-\min \left(\left|e_{0}\right|,\left|e_{1}\right|,\left|e_{2}\right|\right)\right) / 3\right) / 400
$$

where $e_{0}, e_{1}$ and $e_{2}$ are the last game turns evaluations in centipawns. The formula looks complicated, but is easy to understand on one example. If $e_{0}=-420, e_{1}=400$ and $e_{2}=-410$, then the search will stop if the time used is greater than $4 \times((1000-400) / 3) / 400=4 \times 200 / 400=2$ minutes. This is done to prevent spending too much time on already lost or won games.

Condition 3 stops the search if the time already used (in minutes) is greater than:

$$
4 \times\left(10+\max \left(\left|e_{0}+e_{1}\right|,\left|e_{0}-e_{2}\right|,\left|e_{1}+e_{2}\right|\right)\right) / 40
$$

where $e_{0}, e_{1}$ and $e_{2}$ are the last evaluations returned for the last 3 consecutive depths in the current search. For example, if the last 3 evaluations are 53, -63 and 57, then search will stop if the time used is over $4 \times(10+\max (10,4,6)) / 40=4 \times 20 / 40=2$ minutes.

Under these settings, the average time used for finding and analyzing the best two moves was almost exactly 2 minutes, with an average depth of 26 plies.

If the move played in the game is not one of the two best moves already analyzed, it is searched thereafter. The engine is set to analyze only this move, at the exact same depth used for the two best moves. No time limit is set. Usually, searching is fast or very fast, as the hash tables have already been populated during the previous search.

\footnotetext{
${ }^{5}$ Evaluations returned are always relative to the player who is going to move, not to White. So a steady evolution would result in alternating positions evaluations in the game, such as $-40,+41,-42$. Evaluations are always given in centipawns (1 pawn is equal to 100 centipawns).
} 
To enhance further the speed of the search, the game is analyzed in a retrograde way, starting from the end. Thus, the hash tables contain information which also helps in stabilizing the score of the search, and should improve the choices made by the engine.

\subsection{Information saved in the Database}

Evaluation starts only at game turn 10, as the first nine game turns can be considered as opening knowledge ${ }^{6}$. For each position, 2 moves at least are evaluated (the only exception being when there is only one possible move), and sometimes 3 when the move played in the game is not one of the 2 best moves. For each move evaluated, the following elements are recorded:

- the evaluation of the move,

- the depth searched,

- the selected depth searched,

- the number of tablebase hits,

- the time used during the search,

- the average delta between evaluations at $n$ and $n+1$ depth levels,

- the maximal delta between evaluations at $n$ and $n+1$ depth levels and the associated value of $n$.

All this information is saved as comments of the move, and the additional moves are saved as variations with comments.

The headers of each game are limited to the 7 standard PGN tags, plus an Annotator tag which summarizes various information about the game, such as the average time for searching each move, the average depth of the search, the total time used for the game, etc. The database fully complies with the PGN standard, but is however in the simplest mode regarding chess notation: game turns are only indicated by the start and end square and no numbering. This is not a problem for most database programs, and moreover numerous tools exist to convert between PGN formats (such as the excellent pgn-extract program).

Here is an example of the output:

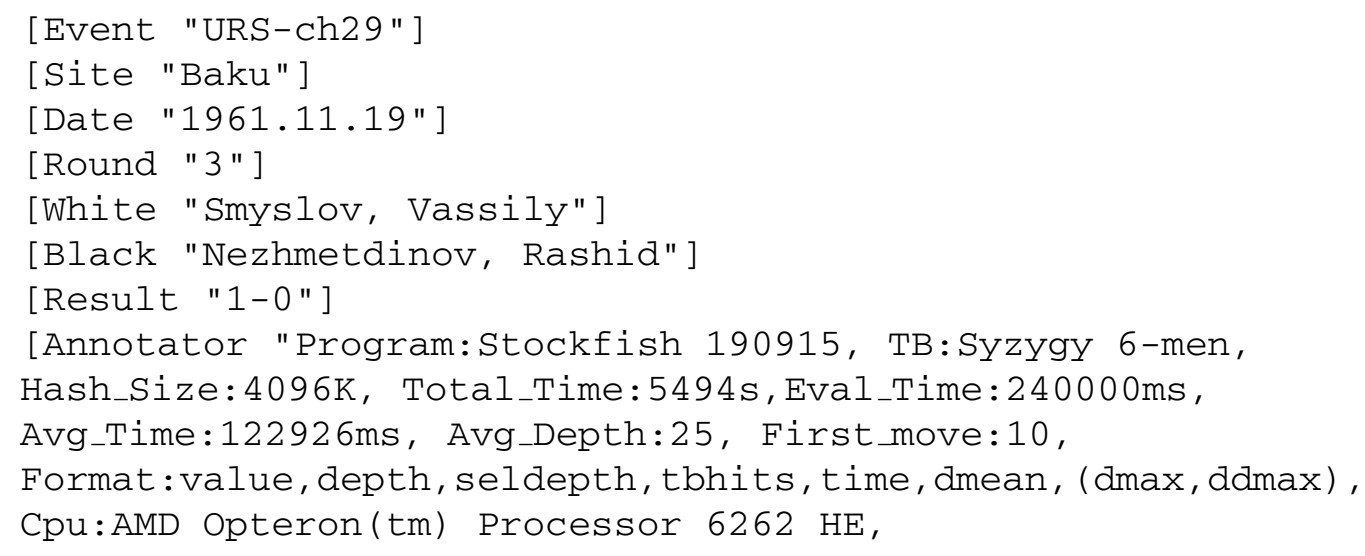

\footnotetext{
${ }^{6}$ Opening knowledge usually goes much deeper than 10 game turns. However, below 10, it is pretty safe to assess that all moves are played "by the book", while this likelihood decreases after. Using 10 (instead of 20 for example) "guarantees" that no mistake made by a player is left unseen, while the only drawback is that the number of "correct" moves for each player will be slightly higher, as long as we believe that opening knowledge is coherent.
} 


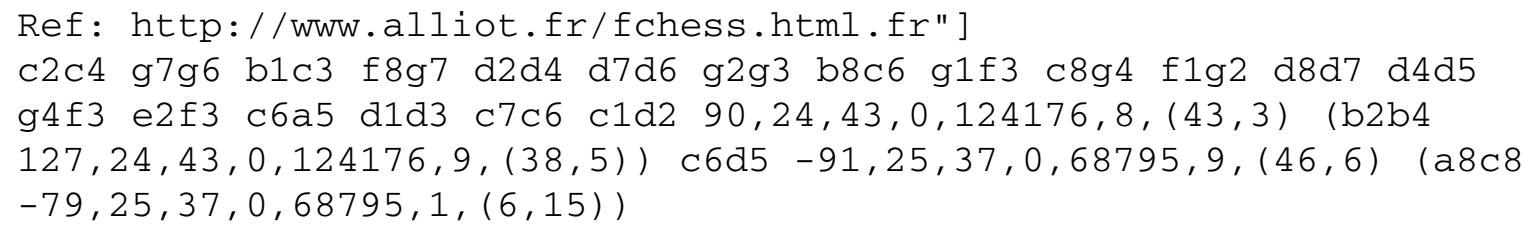

The first move evaluated in the game was $\mathrm{c} 1 \mathrm{~d} 2$, with an evaluation of $90 \mathrm{cp}$ at depth 24 , with a selective depth of 43 , and an evaluation time of $124 \mathrm{~s}$. The mean variation of evaluation along the line was 8 , with the maximal variation being 43 at depth 3. According to STOCKFISH, b2b4 was a better move with an evaluation of $127 \mathrm{cp}$.

\section{INDICATORS CONSIDERED}

Below we consider four different types of indicators. In 3.1 we present three different tactical complexity indicators. In 3.2, we introduce three different conformance indicators. In 3.3 we analyze the notion of distribution of gain. Last, in 3.4, we consider a chess game as a Markovian process.

\subsection{Tactical complexity indicators}

Sullivan (2008) defines a "complexity" indicator for a position which is correlated with the errors made by players.

Here I define three indicators that can be computed from the output of the engine. The correlation of these indicators with the errors made by the players will be evaluated using the classical Pearson's product-moment correlation (Pearson's $\rho$ ).

An experimental evaluation of these indicators is presented in section 4.1.

- Depth of search vs time: A tactical complexity indicator can be computed from the engine depth and time output after analyzing a move. In Fig. 1, the percentage of moves $p(d, t)$ is plotted as a function of depth $d$ reached and of time $t$ used (here $t$ equals 60s, 90s, 120s, 150s, 180s, 210s and 240s) over the 2,000,000 positions analyzed.

The red curve indicates for example that when a position is searched for 240 s then $17 \%$ of the moves are evaluated at depth $26(p(26,240)=0.17)$, and $3 \%$ only at depth 23 or at depth 31 .

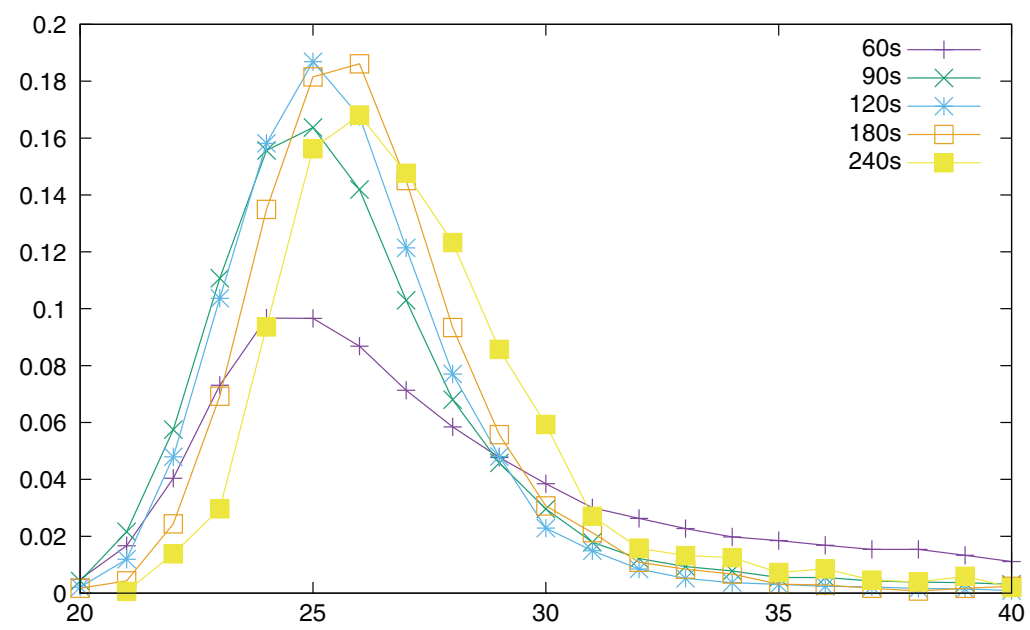

Fig. 1. Percentage of moves as a function of depth reached for a given time. 
Thus if a move is evaluated for 240 s at depth 26 , the position can be considered as average regarding complexity, while it can be considered as a little bit above average complexity if it is evaluated at depth $25(15.5 \%)$ or a little bit below average at depth $27(14.5 \%)$.

Numerous tactical complexity indicators can thus be computed for a move $m$ evaluated at depth $d$ for a time $t$ (these indicators are of course directly correlated with the branching factor of the tree search). If: $p_{\max }(t)=\max _{i} p(i, t)$ and $d_{\max }(t)=\operatorname{argmax}_{i} p(i, t)$ then one of the simplest would be:

$$
\begin{aligned}
C(m) & =\frac{p(d, t)-p_{\max }(t)}{p_{\max }(t)} \text { if } d \leq d_{\max }(t) \\
& =\frac{p_{\max }(t)-p(d, t)}{p_{\max }(t)} \text { if } d \geq d_{\max }(t)
\end{aligned}
$$

- Stability: During the search, the engine saves for each move the mean delta in the evaluation function between two consecutive depths. This can be considered as an evaluation of the stability of the position, and "unstable" positions could be considered as more "complex" than stable ones.

- Unexpected jumps in the evaluation: The engine also saves the largest difference between two successive depths and the depth at which this difference is recorded. This can be seen as a trap in the current position, especially if the jump is large and the depth at which it is recorded is high. Three indicators are computed from these data. The first correlates only the maximal value of the difference, the second correlates only the depth at which the jump in the evaluation appears, and the third one is a product of the 2 values ${ }^{7}$.

\subsection{Move Conformance and Game Conformance}

Below we distinguish between raw conformance (3.2.1), Guid and Bratko conformance (3.2.2) and ponderated conformance (3.2.3).

\subsubsection{Raw conformance}

Every move made by a human player can be compared to the move chosen by the computer program in the same position. The difference between the evaluation $v_{b}$ of the computer program move ${ }^{8}$ and the evaluation $v_{p}$ of the actual move made by the player will be called the raw conformance of the move $\delta=v_{b}-v_{p}$. By construction $\delta$ is always positive.

Some websites ${ }^{9}$ compute similar indicators, and call them Quality of Play. Conformance was chosen as the term bears no presupposition regarding the possible optimality of the move, and also because these indicators measure in fact how much the moves made are similar to the moves that a computer program would play, rather than an hypothetical Quality of Play which is rather difficult to define.

\footnotetext{
${ }^{7}$ These three indicators, especially the third, are very close to the one used by Sullivan. The complete algorithm to compute Sullivan's complexity as described on his website is: (1) the score (call it BEST_SCORE) for the best move (call it BEST_MOVE) is identified and the iteration (call it ITERATION) in which it was so identified is remembered; (2) a new score (call it NEW_SCORE) during a search of depth ITERATION-1 is done for BEST_MOVE; (3) the difference between BEST_SCORE and NEW_SCORE is the raw Complexity score (call it RAW_COMPLEXITY); (4) the Complexity score is $R A W_{-} C O M P L E X I T Y * I T E R A T I O N / 10$. The difference here is that step (2) is not performed, we just use the largest difference between successive evaluations during the search. It would be interesting to modify the system to record enough data to compute Sullivan's complexity indicator.

${ }^{8}$ The computer program move is supposed to be the best possible move, and thus the evaluation of the position is also equal to $y$.

${ }^{9} d b$-chess.com computes for example the STOCKFISH First Choice Ranklist which is, more or less, a conformance 0 index with Guid and Bratko restrictions. However, the details are only available to supporting members of the website.
} 
For a given player, these elementary indicators can be accumulated for a game (which would give a game conformance indicator), or all games played for a year, or all games played during the whole career of the player.

Here the indicator is computed for each player for each move for a given year, and for all years the player was active. For each year, these results are accumulated by intervals of $10 \mathrm{cp}$. Thus $s(P, y, 0)$ is the number of moves played by player $P$ during year $y$ in such a way that the move played has exactly the same evaluation as the move chosen by the computer program. Then $s(P, y, 0.1)$ is the number of moves played in such a way that the raw conformance is between 0 (not included) and $10 \mathrm{cp}$ (or $0.1 \mathrm{p}$ ), subsequently $s(P, y, 0.2)$ is the number of moves played in such a way that the raw conformance is between $0.1 \mathrm{p}$ and $0.2 \mathrm{p}$, and so on.

$R(P, y, \delta)$ defined by:

$$
R(P, y, \delta)=\frac{s(P, y, \delta)}{\sum_{\forall d} s(P, y, d)}
$$

is the percentage of moves belonging to interval $[\delta-0.1, \delta]$ (for $\delta \neq 0$, for $\delta=0$, see above) for player $P$ during year $y$.

$R^{\prime}(P, y, \delta)$ defined by:

$$
R^{\prime}(P, y, \delta)=\frac{\sum_{\forall d \leq \delta} s(P, y, d)}{\sum_{\forall d} s(P, y, d)}
$$

is the percentage of moves played with a conformance $\leq d$.

Last, in order to smooth $R^{\prime}, Q(P, y, \delta)$ is defined by:

$$
Q(P, y, \delta)=\frac{\sum_{\forall j \leq y} 2^{j-y} \sum_{\forall c \leq \delta} s(P, y, c)}{\sum_{\forall j \leq y} 2^{j-y} \sum_{\forall c} s(P, y, c)}
$$

This indicator has a "forgetting factor" over the years: results for year $y-j$ are used to compute the indicator for year $y$ but they count with a factor of $2^{j-y}$ (half for $y-1$, a quarter for $y-2$, etc.).

It would have been interesting to compute these indicators not by years, but by months, with a sliding window. This is however very difficult because some players could spend a lot of time without playing, and moreover the exact date for many old chess events are missing from the database.

We must also notice that this kind of indicator can be defined not for a year, but for only a game and, if the indicator is meaningful, there must be a relationship between the indicator distribution $(R$ is a probability distribution function and $R^{\prime}$ is a cumulative probability distribution function) and the outcome of the game. This is the basis of the validation that will be performed in subsection 4.2.1 for the accumulated conformance (and in subsection 4.3.1 for gain and distribution covariance).

\subsubsection{Guid and Bratko conformance}

In their papers, Guid and Bratko considered an indicator for conformance which was slightly different: they did not take into account the conformance of moves when the evaluation function was already above +200 cp or below -200 cp.

In the rest of this paper this indicator is called Guid and Bratko conformance indicator or sometimes $G \& B$ conformance indicator. 

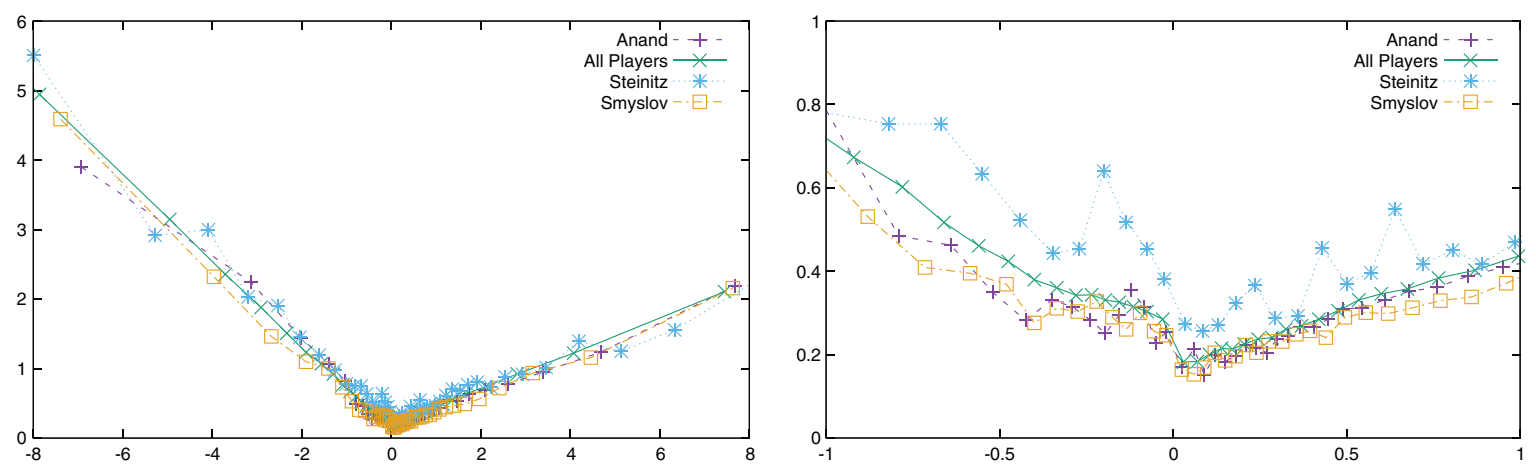

Fig. 2. The distribution of the mean of conformance as a function of the position evaluation for some selected players. The right figure is a zoom of the left one.

As they did not have a large number of games available they only computed this indicator once for each player, aggregating all the games they had for him. However a player's strength changes depending on the tournaments and through the years. So what they computed was not really an indicator of the capacity of a player to find "the right move" (quotes intended), but rather an indicator of his capacity to find the right move during some very specific event(s) in his career. Here, the G\&B indicator is computed as described above for the raw conformance indicator, in order to be able to determine if "cutting out" some moves as advocated by Guid and Bratko is indeed beneficial.

\subsubsection{Ponderated conformance}

As seen above, Guid and Bratko are performing a "hard cut" at [-200;200]. We can see in Fig. 2 the distribution of the mean of the conformance as a function of the evaluation of the position ${ }^{10}$. In this analysis, we are only interested in the moves which have a conformance different from zero, so the latter have been excluded from the statistics. Moreover, moves have been aggregated in order to have statistically significant classes (that is the reason why there are much more points close to 0 , one point represents one class).

The curves of all players are extremely similar, and this is all the most surprising if we consider the "All Players" curve which represent all the players included in the study, i.e., the World Champions and their opponents ${ }^{11}$. Of course most of the players of this study are world class players, as World Champions usually do not play against club players, and the same plot would certainly be different with less strong players. The slope is not the same if $y>0$ or if $y<0$. Players are making bigger mistakes (that might be seen as "desperate maneuvers") when they lose, than when they win. The relationship is not exactly a linear one: when we are close to 0 the positive slope is around 0.2 , while it is 0.25 on the whole interval. The difference is even bigger for the negative slope, with a slope of -0.5 close to 0 and of -0.6 on the whole interval. However, the average of conformance for a position with a valuation of $y$ can be approximated by $\operatorname{avg}(c(y))=a y+b$, with $b=0.18$, and $a=0.26$ for $y>0$ and $b=0.17$ and $a=-0.60$ for $y<0$ (the values are computed for the "All Players" curves).

In order to smooth the cut, a ponderated conformance indicator is defined for each move using the following formula: if $v_{p}$ is the evaluation of the move played and $v_{b}{ }^{12}$ is the evaluation of the best

\footnotetext{
${ }^{10} \mathrm{We}$ only represent here the curves for some selected players for the sake of readability, but I have computed and examined all of them, and they are all similar.

${ }^{11}$ We must however remember that we only plot here the distribution of the mean of the conformance when it is different from 0 , we do not compare the number of times a player makes a "mistake".

${ }^{12} v_{b}$ is always greater than $v_{p}$ by construction.
} 
move then $\delta=v_{b}-v_{p}$ is the conformance of the move, and the ponderated conformance $\delta^{\prime}$ of the move played is given by:

$$
\begin{aligned}
& v_{b} \geq 0: \delta /\left(1+v_{b} / k_{1}\right) \\
& 0>v_{b}: \delta /\left(1+v_{b} / k_{2}\right)
\end{aligned}
$$

The idea is that, while small mistakes made when the evaluation is already very high (or very low) should count for less, they should not be completely discarded. The values of $k_{1}$ and $k_{2}$ can be chosen using the results of the statistical analysis above. If we consider that we map the conformance $\delta$ to a new conformance $\delta^{\prime}\left(v_{b}\right)=\frac{\delta\left(v_{b}\right)}{1+v_{b} / k_{1}}$ then the average value of $\delta^{\prime}\left(v_{b}\right)$ is $\frac{\operatorname{avg}\left(\delta\left(v_{b}\right)\right)}{1+v_{b} / k_{1}}$. But $\operatorname{avg}\left(\delta\left(v_{b}\right)\right)$ is also equal to $a v_{b}+b$. Thus $\operatorname{avg}\left(\delta^{\prime}\left(v_{b}\right)\right)=\frac{a+b v_{b}}{1+v_{b} / k_{1}}=a \frac{1+\frac{b}{a} v_{b}}{1+v_{b} / k_{1}}$. This is equal to $a$ (and is independent of $v_{b}$ ) for $k_{1}=\frac{a}{b}$. Thus we are going to set $k_{1}=0.26 / 0.18=1.44$ and $k_{2}=-0.60 / 0.17=-3.53$.

It is important to stress again why we probably need to ponder $\delta$. The accumulated conformance indicator method (as well as the distribution of gain method described in the next section) take as an hypothesis that an error of $\delta$ has the same influence on the game whatever the evaluation of the position is, and they "aggregate" all these errors in the same class. This is the debatable point: making a small mistake in an already won position seems less decisive than making the same mistake in an equal position ${ }^{13}$. The Markovian interpretation presented in section 3.4 has been specifically designed to avoid this pitfall.

In subsection 4.2.2, I experimentally compare and validate all these indicators by computing their correlation with the outcome of games using Pearson's $\rho$, and we will indeed see that the correlation with the outcome of games is better when pondering $\delta$.

\subsection{Distribution of Gain}

Ferreira (2012) defines the gain of a move in a way which is highly similar to the definition of conformance. He computes the evaluation of the position at game turn $k$ using a fixed depth search, then at game turn $k+1$, and he defines the gain ${ }^{14}$ as $g(k)=v_{b}(k+1)-v_{b}(k)$. If the position evaluation made by the computer was perfect, the gain would be the exact opposite of the raw conformance described above, because the evaluation at game turn $k+1$ should exactly be the evaluation of the move played at position $k$, and thus $g(k)=v_{b}(k+1)-v_{b}(k)=v_{p}(k)-v_{b}(k)=-\delta(k)$. However, mainly because of the monotonicity of the evaluation function discussed above, this is not the case; searching "one move" deeper (because one move has been made) can often increase the value of the evaluation, and thus, while $\delta$ is always positive, $g(k)$ should be negative but is not always. Ferreira's gain method is less "computational intensive", as it just requires to compute one evaluation (the position evaluation) by game turn, instead of computing two (the evaluation of the best move and the evaluation of the move played). However, as discussed above; evaluating two moves instead of one does not multiply the search time by two, and thus it is better in my opinion to define the gain exactly as $\delta$ (disregarding the sign).

Ferreira does not discuss either the problem of "scaling" (or pondering) the gain according to the position evaluation (he only uses "Raw" $\delta$ ), while it is exactly the same problem as discussed above regarding conformance.

\footnotetext{
${ }^{13}$ This is partly again a consequence of the lack of direct mapping of the value of the evaluation function in chess to the probability of winning a game.

${ }^{14}$ Of course, the evaluation of position $P$ is always equal to the evaluation of the best move in position $P$.
} 
Ferreira (2012) interprets conformance as a probability distribution function $R_{P}(\delta)$ which represents the probability for player $P$ to make at each turn a move with conformance $\delta$. This leads to a different definition of the expected value of the result of a game between two players. As player one $(p 1)$ and player 2 ( $p 2$ ) have different distribution functions $R_{p 1}$ and $R_{p 2}$, the probability distribution of the difference between two random variables $R_{p 1}$ and $R_{p 2}$ is the convolution of their distribution $R_{p 1}$ and $R_{p 2}$ :

$$
R_{p 1-p 2}(\delta)=\left(R_{p 2} * R_{p 1}\right)(\delta)=\sum_{m} R_{p 2}(\delta) R_{p 1}(\delta+m)
$$

Here $R_{p 2-p 1}=1-R_{p 1-p 2}$ as it is a probability distribution. Then Ferreira defines the expected gain for $p 1$ in a game between $p 1$ and $p 2$ as the scalar product of the distribution vector with $e=(0, \cdots, 0,0.5,1, \cdots, 1)$, as he interprets $R_{p 1-p 2}(0)$ as a draw, $R_{p 1-p 2}(\delta)$ as a win if $\delta>0$ and as a defeat if $\delta<0$. Then:

$$
s(p 1, p 2)=0 \times \sum_{\delta<0} R_{p 1-p 2}(\delta)+0.5 \times R_{p 1-p 2}(0)+1 \times \sum_{\delta>0} R_{p 1-p 2}(\delta)
$$

Assuming that the contribution of each element of $R_{p 1-p 2}(\delta)$ is the same for all $\delta<0$ (i.e., 0 ) and all $\delta>0$ (i.e., 1) is not obvious. Using a vector with values starting at 0.0 , with a middle value of 0.5 and ending at 1.0, with intermediate values continuously rising feels more intuitive: the contribution of $R_{p 1-p 2}(0.01)$ to the expected result "feels" different from the contribution $R_{p 1-p 2}(10.00)$. We will discuss this problem again in subsection 4.3 when validating experimentally the method.

\subsection{A chess game as a Markovian process}

The indicators described in sections 3.2 and 3.3 are suffering from the problem described at the end of subsection 3.2.3. They basically rely on the idea that an error of $\delta$ in a position $P$ has the same influence on the game whatever the evaluation $v(P)$ of the position is, and they "aggregate" all of them in the same class. Pondering $\delta$ is a way to bend the problem, but the problem is intrinsic to both methods, and bending it is not solving it. Here, I am presenting a method which does not rely on this hypothesis.

If the computer program is performing like an "oracle" always giving the true evaluation of the position and the best possible move, then the database gives a way to interpret chess games for a given player as a Markovian process.

For each position, the computer program is giving us the true evaluation of the position. This evaluation is assumed to remain constant if the best available move is played, while it can only decrease if the player makes a sub-optimal move. The transition matrix, which is triangular ${ }^{15}$, gives for each value of the evaluation function the probability of the value of the evaluation function in the next step.

Table 2 presents this matrix computed with all the games played by Robert James Fischer in 1971. The rows are the value of the evaluation function at state $t$, and the columns are the value of the evaluation function at state $t+1$. Each element in the table is the probability to transition from one state to the other. The sum of all elements in a line is of course equal to 1, and this table defines a right stochastic matrix.

For example, regarding state -0.6 (the evaluation function is between -0.4 and -0.8 ), the probability to remain in state -0.6 (the evaluation function remains between -0.4 and -0.8 ) is $92 \%$, the probability

\footnotetext{
${ }^{15}$ As the computer program is assumed to be a perfect oracle giving the "true" evaluation, the matrix is triangular by construction under this assumption.
} 
Table 2

Transition state matrix for Robert Fischer in 1971 with $g=0.4$,

$$
b_{\text {inf }}=-2.0 \text { and } b_{\text {sup }}=2.0
$$

\begin{tabular}{|r|r|r|r|r|r|r|r|r|r|r|}
\hline & -1.8 & -1.4 & -1.0 & -0.6 & -0.2 & 0.2 & 0.6 & 1.0 & 1.4 & 1.8 \\
\hline \hline-1.8 & 1.00 & 0.00 & 0.00 & 0.00 & 0.00 & 0.00 & 0.00 & 0.00 & 0.00 & 0.00 \\
-1.4 & 0.29 & 0.71 & 0.00 & 0.00 & 0.00 & 0.00 & 0.00 & 0.00 & 0.00 & 0.00 \\
-1.0 & 0.10 & 0.12 & 0.78 & 0.00 & 0.00 & 0.00 & 0.00 & 0.00 & 0.00 & 0.00 \\
-0.6 & 0.01 & 0.01 & 0.06 & 0.92 & 0.00 & 0.00 & 0.00 & 0.00 & 0.00 & 0.00 \\
-0.2 & 0.00 & 0.00 & 0.01 & 0.06 & 0.93 & 0.00 & 0.00 & 0.00 & 0.00 & 0.00 \\
0.2 & 0.00 & 0.00 & 0.00 & 0.00 & 0.14 & 0.86 & 0.00 & 0.00 & 0.00 & 0.00 \\
0.6 & 0.00 & 0.00 & 0.00 & 0.00 & 0.04 & 0.14 & 0.82 & 0.00 & 0.00 & 0.00 \\
1.0 & 0.00 & 0.00 & 0.00 & 0.00 & 0.01 & 0.02 & 0.12 & 0.85 & 0.00 & 0.00 \\
1.4 & 0.00 & 0.00 & 0.00 & 0.00 & 0.00 & 0.01 & 0.01 & 0.10 & 0.88 & 0.00 \\
1.8 & 0.00 & 0.00 & 0.00 & 0.00 & 0.00 & 0.00 & 0.00 & 0.01 & 0.03 & 0.96 \\
\hline
\end{tabular}

to go to state -1.0 (the evaluation function drops between -0.8 and -1.2 ) is $6 \%$, the probability to go to state -1.4 (the evaluation function drops between -0.2 and -1.6 ) is $1 \%$ and the probability to go to state -1.8 (the evaluation function drops below -1.6 ) is also $1 \%$.

State -1.8 is an attractor and can never be left, as the player cannot enhance his position if his opponent is never making a mistake. Diagonal values are the highest, as good players are usually not making mistakes and maintain the value of their evaluation function.

Building this kind of table depends on three parameters, $g$ which is the discretization grain, and $b_{\text {inf }}$ and $b_{\text {sup }}$ which are the bounds outside which a game is supposed to be lost (below $b_{\text {inf }}$ ) or won (above $b_{\text {sup }}$ ).

In the previous table, the evaluation function is considered from the point of view of the player who is going to play, either White and Black. If the evaluation function is considered only from White's point of view, then two tables are built: one for White and one for Black. White's table is the table above; Black's table is easily deduced from White's table using the following formula ${ }^{16}$, where $n$ is the size of the matrix and the array indexes start at 0 :

$$
M_{\text {Black }}(i, j)=M_{\text {White }}(n-1-i, n-1-j)
$$

White's matrix is always triangular inferior, and Black's matrix is triangular superior. Table 3 is the transition matrix for Boris Spassky computed from Black's point of view using all his games in 1971.

Now if $F_{w}$ is Fischer's (White) matrix and $S_{b}$ Spassky's (Black) matrix the product:

$$
M_{F_{w} S_{b}}=F_{w} S_{b}
$$

is the matrix holding the transition probabilities after a sequence of one white move and one black move (probability vectors $v$ are row vectors and will be multiplied from the left, such as in $v M_{F_{w}, S_{b}}=\left(v F_{w}\right) S_{b}$, using the convention of right stochastic matrices). $\mathrm{M}$ is also a stochastic matrix, as it is the product of two stochastic matrices. As such, there exists a vector $\pi$ which is the limit of:

$$
\pi_{n+1}=\pi_{n} M
$$

\footnotetext{
${ }^{16}$ Deducing Black's matrix from White's matrix by symmetry is a perfectly valid idea as long as we think that players play in the same way when they are playing as Black or as White. However, this hypothesis seems to be slightly incorrect as seen in subsection 3.2.3. So it might be beneficial to compute instead two different matrices, one with moves played as Black and one with moves played as White.
} 
Table 3

Black transition state matrix for Boris Spassky in 1971 with $g=0.4$,

$$
b_{\text {inf }}=-2.0 \text { and } b_{\text {sup }}=2.0
$$

\begin{tabular}{|r|r|r|r|r|r|r|r|r|r|c|}
\hline & -1.8 & -1.4 & -1.0 & -0.6 & -0.2 & 0.2 & 0.6 & 1.0 & 1.4 & 1.8 \\
\hline \hline-1.8 & 0.95 & 0.03 & 0.01 & 0.01 & 0.00 & 0.00 & 0.00 & 0.00 & 0.00 & 0.00 \\
-1.4 & 0.00 & 0.77 & 0.11 & 0.04 & 0.00 & 0.08 & 0.00 & 0.00 & 0.00 & 0.00 \\
-1.0 & 0.00 & 0.00 & 0.78 & 0.15 & 0.07 & 0.00 & 0.00 & 0.00 & 0.00 & 0.01 \\
-0.6 & 0.00 & 0.00 & 0.00 & 0.78 & 0.17 & 0.05 & 0.00 & 0.00 & 0.00 & 0.00 \\
-0.2 & 0.00 & 0.00 & 0.00 & 0.00 & 0.79 & 0.20 & 0.01 & 0.00 & 0.00 & 0.00 \\
0.2 & 0.00 & 0.00 & 0.00 & 0.00 & 0.00 & 0.92 & 0.07 & 0.01 & 0.00 & 0.00 \\
0.6 & 0.00 & 0.00 & 0.00 & 0.00 & 0.00 & 0.00 & 0.88 & 0.11 & 0.01 & 0.00 \\
1.0 & 0.00 & 0.00 & 0.00 & 0.00 & 0.00 & 0.00 & 0.00 & 0.80 & 0.12 & 0.08 \\
1.4 & 0.00 & 0.00 & 0.00 & 0.00 & 0.00 & 0.00 & 0.00 & 0.00 & 0.63 & 0.37 \\
1.8 & 0.00 & 0.00 & 0.00 & 0.00 & 0.00 & 0.00 & 0.00 & 0.00 & 0.00 & 1.00 \\
\hline
\end{tabular}

One of the properties of the limit $\pi$ is that it is independent of $\pi_{0}$ as long as $\pi_{0}$ is a stochastic vector (the sum of all elements of $\pi_{0}$ is 1 ), and that it is itself a stochastic vector, called the stationary state of the Markov chain. Instead of calculating the limit, this vector can be easily computed by finding the only stochastic eigenvector associated to eigenvalue 1 .

Using 1971 data from Fischer and Spassky, the stationary vector is:

$$
\pi=(0.07,0.01,0.01,0.04,0.14,0.18,0.07,0.04,0.04,0.40)
$$

The (very) rough interpretation is that the outcome of a match between them should have been $40 \%$ wins for Fischer, 7\% wins for Spassky and 53\% of games drawn ${ }^{17}$. The 1972 World Championship, if Fischer's forfeit in game 2 is removed, ended in $+7=11-2$, or $35 \%$ wins for Fischer, $10 \%$ wins for Spassky and $55 \%$ of games drawn ${ }^{18}$.

\section{FITTING, VALIDATING, COMPARING}

In section $4.1 \mathrm{I}$ am quickly dealing with the complexity indicators presented in the literature and in websites.

These indicators, while interesting, are not as "rich" as the cumulative conformance (4.2), the covariance (4.3) and the Markovian (4.4) indicators; the methodology for these three indicators will mainly be the same: I first check on individual games that the indicator has a good correlation with the outcome of the game, and we try to enhance this correlation by fitting the model to the data. Then I evaluate the indicator not on one game, but on a set of games (here World Championships) to see if "averaging" it on a more macroscopic scale gives coherent results. Then, I compare it to the ELO ranking system, regarding its ability to predict the outcome of games and to rank players. Last I evaluate how they can be used to rank players (which is simple for the accumulated conformance indicator, but not

\footnotetext{
${ }^{17}$ The stationary vector is the limit when time $\rightarrow+\infty$. So the non-extremal values of the eigenvector are the probabilities for the game to end in a draw with unbalanced material. Only games with a stationary evaluation at an extremal position (greater than 1.8 or less than -1.8) can be won (or lost). We are making here the approximation that they are won (or lost), i.e., we suppose that a game whose evaluation ends higher than +1.8 will be won. Technically, the expected value is computed by making the scalar product of the stationary vector $\pi$ with the vector $(0,0.5,0.5 \cdots 0.5,0.5,1.0)$.

${ }^{18}$ Of course, a single example does not give any statistical significance to this indicator. See subsection 4.4.3.
} 
so simple for the other two). To do this, I am going to use the World Championships for which the complete data for the two players are available ${ }^{19}$, and I am going to compute the three indicators for the year just before the championship, using a "forgetting factor" as described in section 3.2. I will then use these indicators to compute the predicted result of the championship, and I will compare it to the actual result and to the predicted outcome compute with the ELO model (when ELO rankings exist).

A quick reminder might be useful here; the ELO ranking system was designed, from the start, to be able to estimate the probability of the outcome of a game between two players, and in this system estimating the outcome and ranking players is intimately linked as they both depend on each other: points are added (respectively subtracted) when you defeat a player who has a better ranking (respectively when you lose against a player with a lesser ranking), and the rankings are used to estimate the expected outcome of a game. There is no such relationship for intrinsic indicators. One advantage of the intrinsic predictors is that, as soon as they have been computed, they enable to compare any players even if they belong to completely different periods. They are only based on the conformance of moves (the "quality of play" is intrinsic to a player) and are thus completely independent of the possible "drifting through years" problem of the ELO indicator.

\subsection{Complexity indicators}

I present in Table 4 the correlations between the magnitude of the error made by the player with the following indicators.

D/t: Depth vs Time: describes complexity as a function of the depth reached regarding time use to reach it.

Stab: Stability: depends on the mean delta in the evaluation function between two consecutive depths in the search (see section 3.1).

JumpV: Jump Value: depends on the largest difference in the evaluation function between two successive depths in the search.

JumpD: Jump Depth: depends on the depth where the difference between two successive evaluations are the largest.

JD x JV: Jump Depth times Jump Value: product of the previous two indicators. (see section 3.1).

The correlations were computed using Pearson's $\rho^{20}$.

These indicators were computed for all the moves played by each World Champion, and were also aggregated for all moves played by all World Chess Champions (the Champs line). They were also computed for all the moves of all the games present in the database (the All line). The Others line is the complement of the All line and the Champs line (i.e., all moves present in the database played by players who were not World Champions).

\footnotetext{
${ }^{19}$ This means that both of them have been at least once World Champions, as we only have all data for players who have been World Champion. To be able to predict scores for all World Championships, all the games of all players who played once in a World Championship would have to be added to the database. This could be the subject of a later study. Moreover, some players do not have active matrices for the year before their World Championship, so these championships were not taken into account either.

${ }^{20}$ Pearson's $\rho$ is the covariance of the two variables divided by the product of their standard deviations. The possible values range from -1 to $+1 .-1$ is a perfect negative linear correlation, +1 a perfect positive linear correlation and 0 represents no linear correlation at all.
} 
Table 4

Correlations of complexity indicators: Depth vs time,

Stability, Jump Value, Jump Depth and a composite of Jump Value and Jump Depth

\begin{tabular}{|l|r|r|r|r|r|}
\hline Name & D/t & Stab & JumpV & JumpD & JD x JV \\
\hline Steinitz & 0.092 & 0.327 & 0.349 & 0.176 & 0.361 \\
Lasker & 0.046 & 0.235 & 0.296 & 0.147 & 0.306 \\
Capablanca & 0.081 & 0.355 & 0.417 & 0.149 & 0.432 \\
Alekhine & 0.064 & 0.282 & 0.315 & 0.185 & 0.343 \\
Euwe & 0.046 & 0.220 & 0.306 & 0.141 & 0.311 \\
Botvinnik & 0.071 & 0.333 & 0.427 & 0.128 & 0.439 \\
Smyslov & 0.035 & 0.189 & 0.233 & 0.123 & 0.223 \\
Tal & 0.058 & 0.256 & 0.311 & 0.129 & 0.290 \\
Petrosian & 0.044 & 0.241 & 0.285 & 0.110 & 0.300 \\
Spassky & 0.044 & 0.270 & 0.301 & 0.136 & 0.314 \\
Fischer & 0.011 & 0.273 & 0.310 & 0.134 & 0.313 \\
Karpov & 0.046 & 0.216 & 0.264 & 0.122 & 0.270 \\
Kasparov & 0.058 & 0.313 & 0.384 & 0.128 & 0.385 \\
Khalifman & 0.074 & 0.258 & 0.317 & 0.138 & 0.347 \\
Anand & 0.057 & 0.243 & 0.329 & 0.131 & 0.344 \\
Ponomariov & 0.048 & 0.156 & 0.174 & 0.150 & 0.168 \\
Kasimdzhanov & 0.056 & 0.340 & 0.405 & 0.106 & 0.350 \\
Topalov & 0.036 & 0.223 & 0.252 & 0.135 & 0.276 \\
Kramnik & 0.056 & 0.253 & 0.290 & 0.148 & 0.307 \\
Carlsen & 0.061 & 0.252 & 0.319 & 0.125 & 0.295 \\
\hline Champs & 0.053 & 0.254 & 0.308 & 0.135 & 0.312 \\
Others & 0.031 & 0.104 & 0.104 & 0.138 & 0.101 \\
All & 0.038 & 0.161 & 0.185 & 0.135 & 0.180 \\
\hline \hline
\end{tabular}

The first thing to notice is the fact that the $D / t$ indicator is almost not significant. The correlation is extremely low, even if it is always positive, for all players. Apparently, the branching factor of the tree does not seem to be a very good indicator of what some authors call "the complexity" of the position. However, there is no indicator which is extremely significant. The best one seems to be the composite JumpxDepth indicator, which is equal to 0.312 for World Champions, while it is only 0.101 for the other players. The most plausible interpretation is that World Champions usually play the "right moves" when the positions are stable, and make mostly mistakes in unstable positions, while "ordinary" players are more prone to make mistakes in all kind of positions. The only players having an indicator over 0.4 are Botvinnik and Capablanca, which were famous for their positional and consistent play.

A lesson to learn from these indicators is probably that on the one hand, it would be interesting to collect and save more data during the search, such as the value of the evaluation for all depths of the search (and not only the mean and the max), to try to compute other indicators, as the ones computed here, while interesting, do not seem to carry an extremely high significance. On the other hand, it is also possible that there is no such thing as a simple "complexity indicator" of a position that could be correlated with the errors made by the players, and that the complexity of the position depends on many other, less evident, factors.

\subsection{Cumulative conformance}

The cumulative conformance section is partitioned into four subsections: correlation with the outcome of a game (4.2.1), conformance of play in World Championships (4.2.2), conformance of play during a whole career (4.2.3) and predicting the results of World Championships matches (4.2.4). 


\subsubsection{Correlation between cumulative conformance and the outcome of one game}

In section 3.2 I have defined different possible indicators regarding the conformance of moves. Below, I am going to correlate these indicators to the outcome of games using again Pearson's $\rho$.

First, it is interesting to have an idea of the distribution of the conformance for all the positions evaluated during this study. We only keep positions after game turn 10 and positions where the move to play is not forced. This leaves around 1,600,000 positions (respectively 1,350,000 for Guid and Bratko who eliminate positions with an evaluation lower than -2.00 or higher than 2.00). The conformance is equal to 0 for 980,000 moves (respectively 842,000), which is a large majority. In Fig. 3 the number of positions for each conformance, up to 1.99, is plotted (conformance is measured in centipawns, so it starts at 0.01 and goes up to 1.99 by 0.01 steps). The class after 1.99 , which is not plotted, contains all positions with a conformance greater than 2.00; there are around 53000 such positions.

For each game and each type of conformance, three different kinds of conformance (as defined in section 3.2) are computed. We quickly summarize them below.

- Raw conformance $\delta=v_{b}-v_{p}$ is just the raw difference between the evaluation $v_{b}$ of the best move and the evaluation $v_{p}$ of the move made by the player.

- Guid and Bratko conformance is defined in a similar way, but the positions with an evaluation higher than +2 or lower than -2 are not considered.

- Ponderated conformance is defined by $\delta^{\prime}=\delta /\left(1+v_{b} / k_{1}\right)$ for $v_{b}>0$ and $\delta^{\prime}=\delta /\left(1+v_{b} / k_{2}\right)$ for $v_{b}<0$, where $k_{1}$ and $k_{2}$ are suitable constants. In subsection 3.2.3, after a statistical analysis of the distribution of errors, $k_{1}=1.44$ and $k_{2}=-3.53$ are chosen.

In the rest of this section, each time the word "conformance" is used, it can represent any of these three meanings, except when explicitly stated otherwise. We are interested in the cumulative conformance for White (respectively Black) during one game defined by $p_{w}(x)$ (respectively $p_{b}(x)$ ):

$$
\begin{aligned}
p_{w}(x) & =\frac{n b \_m o v e s \_w h i t e(\delta \leq x)}{\text { total_moves_white }} \\
p_{b}(x) & =\frac{n b \_m o v e s \_b l a c k(\delta \leq x)}{\text { total_moves_black}}
\end{aligned}
$$

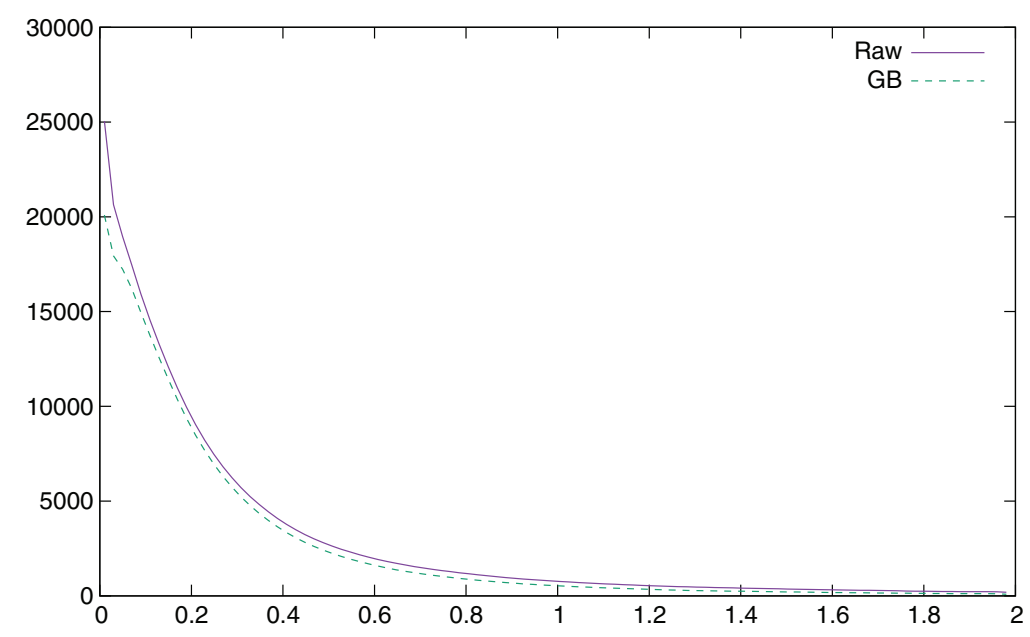

Fig. 3. Distribution of conformance, excluding first and last class. 


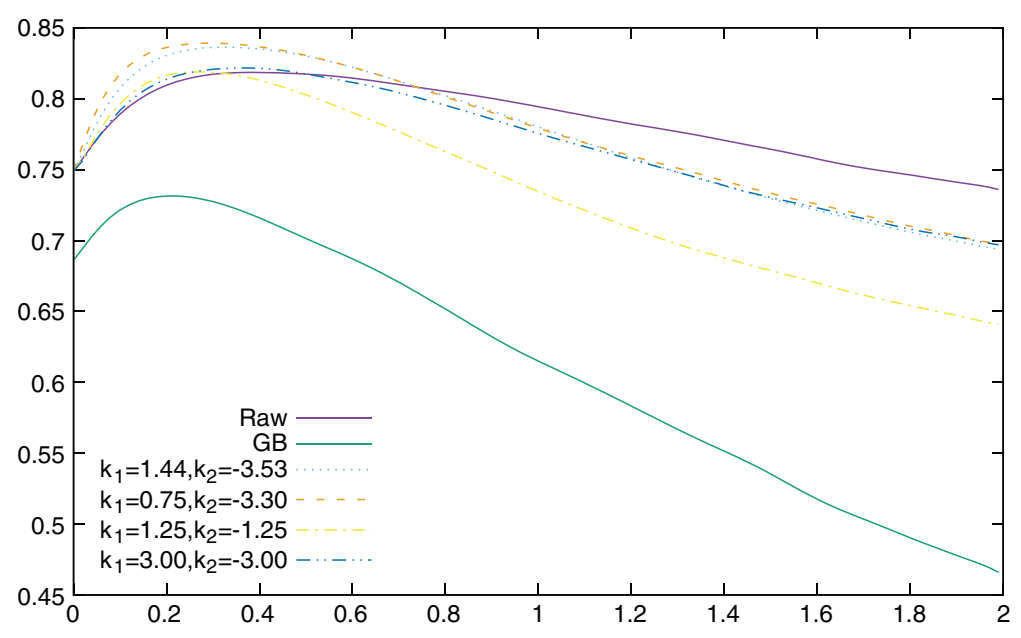

Fig. 4. Correlation of accumulated conformance indicators for raw conformance, G\&B conformance and different values of $k_{1}$ and $k_{2}$ for ponderated conformance.

total_moves_white (respectively Black) is the total number of white moves in the game which are taken into account: this value is simply the number of white moves in this game minus the opening moves and minus the moves which are forced (there is only one move possible $)^{21}$. nb_moves_white $(\delta \leq x)$ (respectively Black) is the number of moves with a conformance less than or equal to $x$, taken only in the moves taken into account as defined above.

Then $p(x)=p_{w}(x)-p_{b}(x)$ is the difference between White's and Black's conformance for a given game. There are around 26,000 games, and thus 26,000 $p(x)$ for each $x$. Now, we wish to know for which value of $x p(x)$ has the best correlation with the outcome of the game. Thus, for each $x$ we compute Pearson's $\rho$ by correlating for each $x$ the 26,000 $p(x)$ with the outcome of the 26,000 corresponding games $(+1$ if White wins, 0 for a draw and -1 if White loses). An optimization was quickly performed using a Nelder and Mead (1965) simplex algorithm ${ }^{22}$ to find the best correlation possible, and the optimal values found are $k_{1}=0.75$ and $k_{2}=-3.3$.

Figure 4 represents the correlations of the accumulated conformance indicators starting at conformance 0 . The best correlation is found for $d \leq 0.3$ for the raw and ponderated conformances, and for $d \leq 0.2$ for the G\&B conformance. It is interesting to notice that the choices made for $k_{1}=1.44$ and $k_{2}=$ -3.53 in subsection 3.2.3 work remarkably well when compared to the optimal curve $k_{1}=0.75$ and $k_{2}=-3.30$. The decision to use two different slopes depending on the sign of the evaluation function is also validated when we compare the previous curves to the curves defined by $k_{1}=-k_{2}=1.25$ and $k_{1}=-k_{2}=3.00$.

It is important to try to understand why there is a "bump" in the curve representing correlation (i.e., why the optimal correlation is reached around $\delta \leq 0.30$ and not somewhere else). My interpretation is the following: having a better conformance for "perfect" $(d=0)$ moves is of course extremely important because the "perfect" moves class is by far the largest and overshadows the others. However, having a better conformance here does not tell us anything about the distribution of the other moves, and even if there are less moves in the other classes, there are still some of them, especially in the class closest to 0 . Thus "adding" those classes to the conformance indicator gives more information about

\footnotetext{
${ }^{21}$ For Guid and Bratko conformance, all positions with an evaluation over 2.0 or below -2.0 are also removed.

${ }^{22} \mathrm{We}$ are making the assumption that the function is locally convex around the optimum, which is quite reasonable here.
} 
the distribution of the moves and "captures" important information. However, after a point, adding new classes which contain a small number of moves adds less meaningful information, and the correlation decreases.

There is still an other point to discuss: how is the outcome of the game correlated to the mistakes made, in other words what happens when we correlate the outcome of the game to $p^{\prime}(x)$ defined by

$$
\begin{aligned}
p_{w}^{\prime}(x) & =\frac{n b \_m o v e s \_w h i t e(\delta \geq x)}{\text { total_moves_white }} \\
p_{b}^{\prime}(x) & =\frac{n b \_m o v e s \_b l a c k(\delta \geq x)}{\text { total_moves_black }} \\
p^{\prime}(x) & =p_{w}^{\prime}(x)-p_{b}^{\prime}(x)
\end{aligned}
$$

First, let us notice that $n b_{\_} m o v e s_{-} w h i t e(\delta \geq x)+n b_{-} m o v e s_{-} w h i t e(\delta \leq x)=$ total_moves_white. So:

$$
\begin{aligned}
p_{w}^{\prime}(x) & =\frac{n b \_m o v e s \_w h i t e(\delta \geq x)}{\text { total_moves_white }} \\
& =\frac{\text { total_moves_white }-n b \_m o v e s \_w h i t e(\delta \leq x)}{\text { total_moves_white }} \\
& =1-\frac{n b \_m o v e s \_w h i t e(\delta \leq x)}{\text { total_moves_white }} \\
& =1-p_{w}(x)
\end{aligned}
$$

Thus Pearson's $\rho$ for $p^{\prime}(x)$ is ${ }^{23}-\rho(p(x))$. Thus the curve representing the correlation of $p^{\prime}(x)$ will be exactly the opposite of the one of $p(x)$, with the same extrema at the same positions.

This result might seem paradoxical. Intuitively, we might think that making big errors should be quite strongly correlated to the result of the game. This is of course true: in Fig. 13 in subsection 4.4.1 we will see that the result of the game is very strongly correlated to the highest evaluation reached in the game. But here the accumulated conformance indicator(s) is not measuring this kind of correlation. Accumulated conformance is in fact measuring the combination of two things at the same time: on the one hand, it has to take into account how often a player is losing a game when he ${ }^{24}$ is making a (big) mistake, but it also depends on the probability of making big mistakes. A player who loses always when making a 50cp mistake, but only makes such mistakes one game out of one hundred will lose less often than a player who never loses games when he makes a 50cp error, and loses them only when he makes a 100cp error, but makes such mistakes one game out of fifty.

It is important to remember that I have only be maximizing the correlation of the difference of the accumulated conformance indicator with the result of the game, which is not the same thing as "fitting" the value of the difference of the conformance between two players with the result of the game. As Pearson's $\rho$ is invariant under linear scaling, it is possible using a classical least square method to find $\alpha$ and $\beta$ such as $r=\beta d+\alpha$ is the best approximation of the actual result of the game (here $d$ stands for the difference of the conformance indicators of the two players). This will of course not change Pearson's $\rho$, so this computation can be done independently of the optimization of $k_{1}$ and $k_{2}$, and we

\footnotetext{
${ }^{23}$ Pearson's $\rho$ is semi-invariant under affine linear transformations, i.e., $\rho(a x+b)=\operatorname{sgn}(a) \rho(x)$.

${ }^{24}$ For brevity, we use "he" and "him" whenever "he or she" and "him or her" are meant.
} 

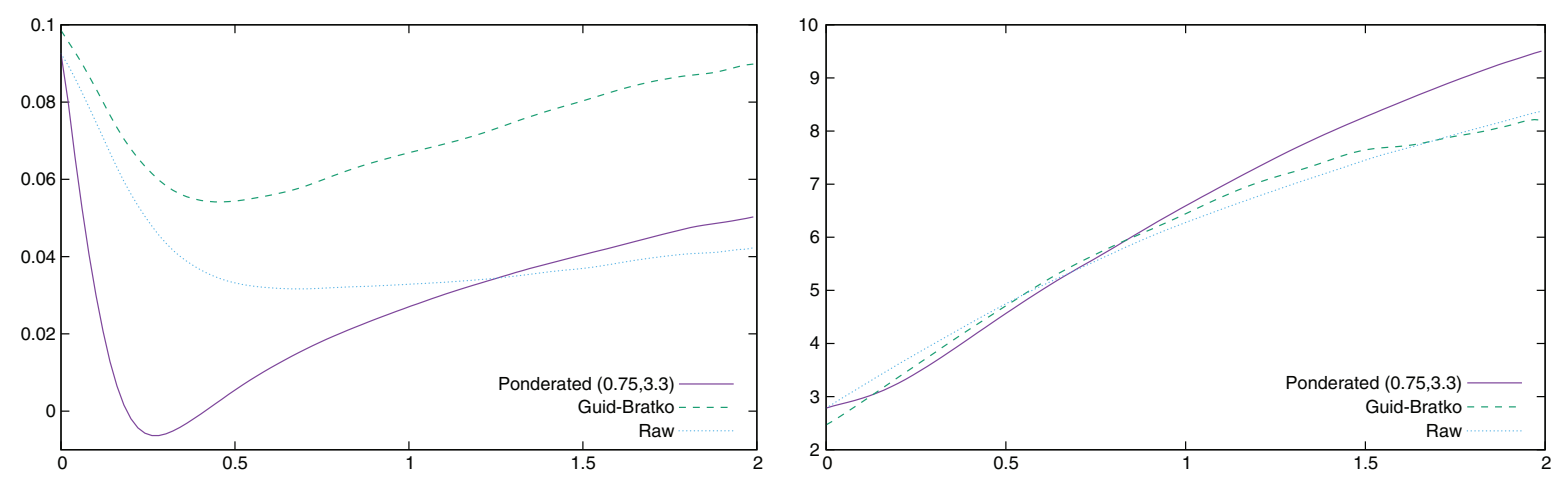

Fig. 5. $\alpha$ (left) and $\beta$ (right) values as a function of the difference of the accumulated conformance indicators of the two players.

can compute $\alpha$ and $\beta$ for all possible values of $x$ such as $\delta \leq x$. We expect ${ }^{25} \alpha$ to be rather close to 0 , while $\beta$ should increase with $x$.

In Fig. 5 we have plotted the values of $\beta$ and $\alpha$ as a function of $x$. Let us remember that the optimal value of $x$ is 0.3 for ponderated and raw conformance, and 0.2 for Guid and Bratko conformance; the optimal values of $(\alpha, \beta)$ are: $\operatorname{Raw}\left(\alpha=4.310^{-2}, \beta=4.00\right)$, Guid and Bratko $\left(\alpha=6.710^{-2}, \beta=\right.$ 3.37), and Ponderated ( $\alpha=-7.010^{-3}, \beta=3.64$ ). The values of $\alpha$ show that there is a small positive bias regarding raw conformance (and Guid and Bratko conformance). The correlation has always been computed by subtracting Black's value from White's value, so this shows that, for identical raw values of the conformance indicator, White wins more often than Black ${ }^{26}$. A quick statistical analysis of the 26,000 games shows that the average score of a game is 0.12 (White is winning $56 \%$ of the points). It is common knowledge that, in chess, White wins slightly more often than Black, and the usual explanation is that White's positions are usually "better" as White plays first. This explanation is of course correct $^{27}$, but there might be another factor.

When plotting the difference of the raw accumulated conformance indicator for White and for Black, it is always positive (see left part of Fig. 6). White is playing 61.1\% perfect moves $(x=0)$, while Black is only playing $60.2 \%$ perfect moves. The difference even rises for larger $x$ and is maximal around $x=0.25$ where it reaches almost $2 \%$. So, Black is in a way, making more mistakes than White. Why it is so is more difficult to interpret. We have already seen (subsection 3.2.3) that players are making more serious mistakes when they are in unfavorable positions; as Black is usually starting with a slight disadvantage, the same kind of psychological bias might encourage them to take more risks, and thus to make more mistakes. On the right side of Fig. 6, we see that the distributions of White's and Black's conformance are different. White is performing better at 0 and slightly above, while Black is better below 0 . This figure also confirms that while the level of play remains consistent when the evaluation of the position is positive, it is degrading fast for negative ones. We also understand why ponderated conformance corrects the bias: it is "stretching" differently the positive and the negative side of the curve because it is using two different constants to "bend" the distributions. The fact that the difference

\footnotetext{
${ }^{25}$ The output of games used for computing the correlation was $-1 / 0 /+1$, not $0 / 0.5 / 1$, which does not change Pearson's $\rho$ either, as it is also invariant under linear scaling of the value being correlated

${ }^{26}$ On the opposite, the ponderated conformance corrects the bias almost perfectly ( $\alpha \simeq 0$ for $x=0.3$ ), which is explained later.

${ }^{27}$ Plotting the position evaluations reached by White and Black shows that they follow an almost normal distribution, but White's distribution is centered slightly over 0 , while Black's distribution is centered below 0 , and plotting them as a function of the move number shows that Black usually starts in an inferior position.
} 

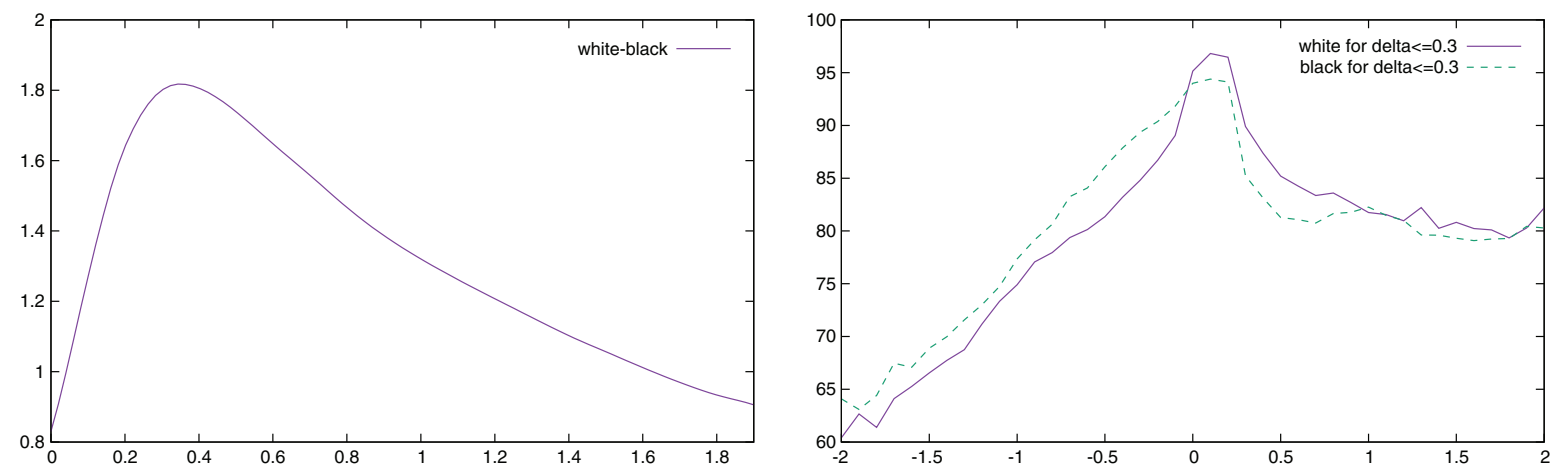

Fig. 6. Difference between the accumulated raw conformance indicator of White and Black (in percent) as a function of $\delta$ (left), and percentage of moves with an accumulated raw conformance $\delta \leq 0.3$ as a function of the position evaluation (right).

between White and Black is maximal around $x=0.25$ might be another reason why the accumulated conformance indicator has the best correlation around this value.

In conclusion, the advantage of the accumulated conformance indicator is that it is a scalar, and it is thus easy to consider it as a ranking. The player with the best indicator is just supposed to be the best player. However, this discussion should remind us that cumulative conformance is not a beast which is easily tamed, and it is much more difficult to interpret it than it might seem at first glance. A second important thing to remember is that we have "fitted" the model to the data using only games played by world class champions; it is extremely possible that results and parameters would be different for club players, as the distribution of their moves is very different; thus some classes with high $\delta$ which are marginal here could have a much higher importance.

\subsubsection{Conformance of play in World Championships}

In this subsection we are working on many games at once. The conformance is computed for all the moves in all these games at the same time; we are using here World Championships games, in the same way as the previous work by Guid and Bratko concentrated exclusively on these games.

The left part of Fig. 7 gives for each championship since 1886(1) the actual result, (2) the expected result using the accumulated conformance indicator and (3) the expected result using simply the percentage of "perfect moves" (appropriate $\alpha$ and $\beta$ as defined in the section above are used to scale properly the indicator). The number of games, or the time controls were not identical for all these events, but they were mainly similar. The results for the FIDE World Championships played in k.o. mode from 1998 to 2004 are not taken into account, as these time controls were criticized for lowering the quality of play.

The correlation of the actual result with the indicators is adequate, but visually it is not so clear that the ponderated conformance is much better than the simple "perfect move" percentage. The ponderated conformance is usually closer to the actual result, which is often overestimated by the "perfect move" percentage. However, the ponderated conformance sometimes "misses" results, such as the result of the last WCH (Carlsen-Anand 2013), which is grossly underestimated.

In the right part of Fig. 7, we plot the difference in conformance between the two opponents for four World Championships $^{28}$. This curve tells us why ponderated conformance at $\delta \leq 0.3$ is partly missing

\footnotetext{
${ }^{28}$ It is impossible to print in this article all the results available for all players and all World Championships. These results will however be made freely available online, along with the full database.
} 

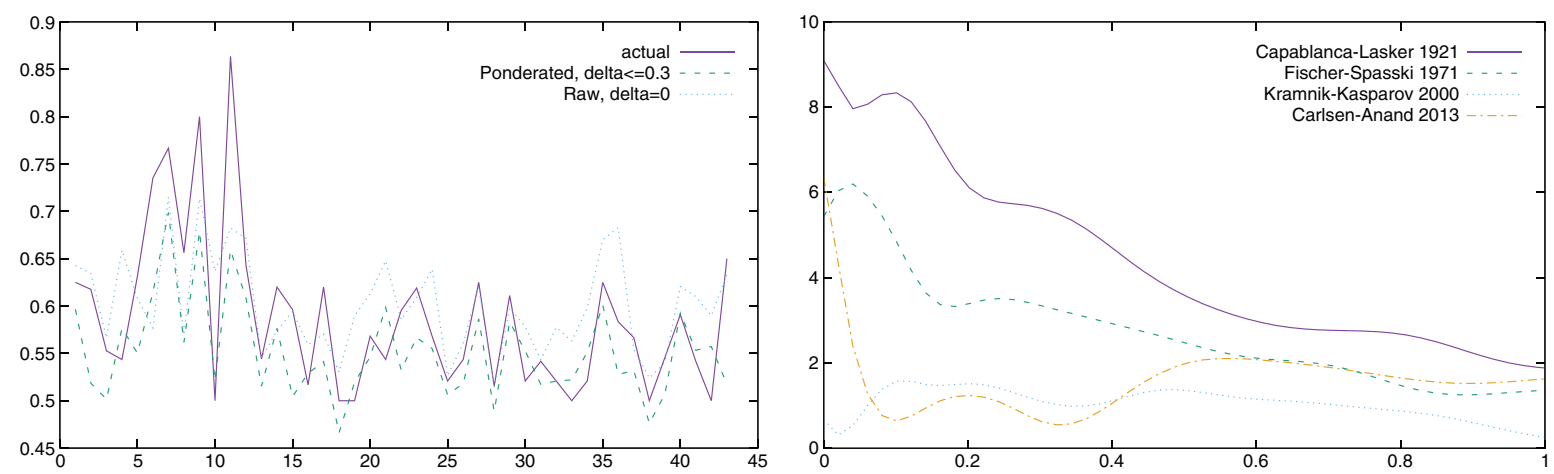

Fig. 7. Actual score and expected scores for all World Championships since 1886 (left) and difference of conformance between two opponents for four World Championships.
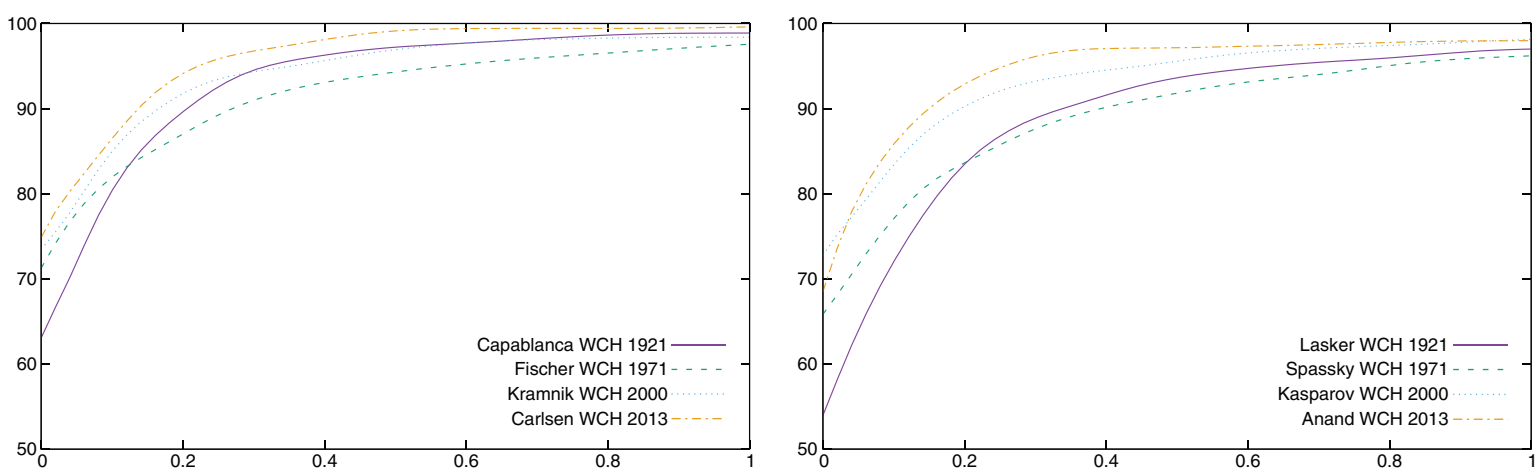

Fig. 8. Performance of winners (left) and losers (right) in four World Championships.

its target for the 2013 Championship. The difference between Carlsen and Anand is extremely high for $\delta=0$ and then falls steeply, and is small around $\delta \leq 0.3$. A careful visual study of all the curves for the 41 World Championship hints to a possible interpretation; it looks like the result depends first on the difference of the indicator for $\delta=0$. However, if this difference becomes "small", then the result seems to be determined by the difference for higher values of $\delta$. This remark has to be taken with extreme caution and requires further investigation, but it is not impossible, as this indicator is an aggregator, and its interpretation is complex.

In Fig. 8 we plot the performance of winners (left) and losers (right) during these four WCH. The performance by José Raul Capablanca in 1921 is definitely remarkable ${ }^{29}: 63 \%$ of his moves were exactly those chosen by the computer ( $0 \mathrm{cp}), 81 \%$ were at a score less than $10 \mathrm{cp}$ of the move chosen, $90 \%$ at a score less than $20 \mathrm{cp}$ and $95 \%$ at a score less than $30 \mathrm{cp}$. It took years to find other players able to perform so well in a WCH. It is however interesting to notice that the "conformance" of players has steadily raised. In 2013, Magnus Carlsen scored respectively $75 \%$ at $0 \mathrm{cp}, 86 \%$ at $10 \mathrm{cp}, 95 \%$ at $20 \mathrm{cp}$ and $97 \%$ at 30cp. For all championships from 2000 to 2013, all winners scored better than Capablanca at $0 \mathrm{cp}$, and most of them scored better at 10cp, 20cp and 30cp. Kasparov lost the $2000 \mathrm{WCH}$ while his performance was his best ever in a WCH, Kramnik was simply better.

\footnotetext{
${ }^{29}$ It is however useful to remember that the 1921 match against Lasker lasted only 11 games: Lasker was not at the top of his form and was completely outperformed by Capablanca; the difference is one of the largest computed.
} 
Table 5

Accumulated conformance predicted score $\left(A_{C_{S}}\right)$, Covariance predicted score $\left(C_{O V_{S}}\right)$, Markovian predicted scores $\left(M_{S}\right)$, actual scores $\left(A_{S}\right)$ and ELO predicted scores $\left(E L O_{S}\right)$ when available for World Championships

\begin{tabular}{|c|c|c|c|c|c|}
\hline Championship & $A_{C_{S}}$ & $C_{O V_{S}}$ & $M_{S}$ & $A_{S}$ & $E L O_{S}$ \\
\hline Euwe-Alekhine 1935 & $57 \%$ & $60 \%$ & $61 \%$ & $52 \%$ & \\
\hline Alekhine-Euwe 1937 & $53 \%$ & $51 \%$ & $57 \%$ & $62 \%$ & \\
\hline Smyslov-Botvinnik 1957 & $50 \%$ & $49 \%$ & $51 \%$ & $56 \%$ & \\
\hline Botvinnik-Smyslov 1958 & $45 \%$ & $48 \%$ & $49 \%$ & $54 \%$ & \\
\hline Botvinnik-Tal 1961 & $49 \%$ & $51 \%$ & $52 \%$ & $59 \%$ & \\
\hline Petrosian-Botvinnik 1963 & $51 \%$ & $58 \%$ & $57 \%$ & $57 \%$ & \\
\hline Petrosian(2660)-Spassky(2670) 1966 & $49 \%$ & $65 \%$ & $45 \%$ & $52 \%$ & $48 \%$ \\
\hline Spassky(2690)-Petrosian(2650) 1969 & $48 \%$ & $33 \%$ & $54 \%$ & $54 \%$ & $56 \%$ \\
\hline Fischer(2785)-Spassky(2660) 1972 & $54 \%$ & $53 \%$ & $63 \%$ & $63 \%$ & $67 \%$ \\
\hline Kasparov(2710)-Karpov(2700) 1985 & $47 \%$ & $46 \%$ & $53 \%$ & $54 \%$ & $51 \%$ \\
\hline Kasparov(2710)-Karpov(2700) 1986 & $50 \%$ & $51 \%$ & $51 \%$ & $53 \%$ & $51 \%$ \\
\hline Kasparov(2720)-Karpov(2720) 1987 & $48 \%$ & $48 \%$ & $48 \%$ & $50 \%$ & $50 \%$ \\
\hline Kasparov(2770)-Karpov(2710) 1990 & $53 \%$ & $55 \%$ & $54 \%$ & $52 \%$ & $59 \%$ \\
\hline Kasparov(2820)-Anand(2720) 1995 & $51 \%$ & $54 \%$ & $50 \%$ & $58 \%$ & $64 \%$ \\
\hline Kramnik(2730)-Kasparov(2810) 2000 & $51 \%$ & $48 \%$ & $59 \%$ & $57 \%$ & $39 \%$ \\
\hline Anand(2800)-Kramnik(2785) 2008 & $50 \%$ & $42 \%$ & $52 \%$ & $54 \%$ & $52 \%$ \\
\hline Carlsen(2840)-Anand(2780) 2013 & $54 \%$ & $54 \%$ & $60 \%$ & $65 \%$ & $58 \%$ \\
\hline
\end{tabular}

\subsubsection{Whole career}

Figures 16 and 17 display the conformance indicator for all World Champions for their whole career, respectively for $d=0$ (Fig. 16) and $d \leq 0.3$ (Fig. 17). Players perform differently depending on the bound set on move conformance. For example, Fischer has outstanding records for $d=0$, while his performances for $d \leq 0.3$ are more ordinary ${ }^{30}$.

\subsubsection{Predicting the results of World Championships}

Below we compare the score predicted for World Championships by the accumulated conformance predictor $\left(A_{C_{S}}\right)$ to $(1)$ the actual score $\left(A_{S}\right)$ and to $(2)$ the score predicted using ELO tables $\left(E L O_{S}\right)$. This indicator can only be computed for the World Championships where both players were at least once World Champion, because only World Champions have all their games evaluated. The available results are presented in Table 5 in the $A_{C_{S}}$ column. Column $A_{S}$ contains the actual score of the WCH and $E L O_{S}$ the predicted result of the championship according to the ELO ranking of both players when it was available (column $C_{O V_{S}}$ contains covariance predicted score and column $M_{S}$ Markovian predicted scores, see subsections 4.3.3 and 4.4.3). The accumulated conformance predictor $A_{C_{S}}$ is computed by taking the result of the games played by both players the year before the $\mathrm{WCH}$ and applying the parameters giving the best correlation $\left(\delta=0.3, \alpha=-0.007, \beta=3.64, k_{1}=0.75, k_{2}=3.3\right)$.

For the 11 World Championships for which the ELO prediction is available, the mean difference between the actual score and the ELO predicted score is 5\%. For the accumulated conformance predictor, the mean difference between the actual score and the accumulated conformance predicted

\footnotetext{
${ }^{30}$ From a close examination of all the curves and all the results for all World Championships (not presented here) a possible interpretation regarding the outcome of the game is that the difference for $d=0$ is the most important regarding the outcome of a match if this difference is large. However, when this difference is small, it looks like the difference for $d \leq 0.3$ becomes more important. If this interpretation is correct, then Robert Fischer certainly dominated chess in his own time.
} 
Table 6

Statistical results for the covariance indicator

\begin{tabular}{|l||r|r|r|r|r|r|}
\hline & Raw & G\&B & $\begin{array}{r}k_{1}=1.44 \\
k_{2}=-3.53\end{array}$ & $\begin{array}{r}k_{1}=0.37 \\
k_{2}=-3.70\end{array}$ & $\begin{array}{r}k_{1}=1.20 \\
k_{2}=-3.41 \\
s=1.16\end{array}$ & $\begin{array}{r}k_{1}=0.82 \\
k_{2}=-2.37 \\
\text { Spline }\end{array}$ \\
\hline$\rho$ & 0.806 & 0.749 & 0.817 & 0.825 & 0.875 & 0.879 \\
\hline $\bar{x}$ & 0.012 & 0.010 & 0.018 & 0.031 & 0.020 & 0.017 \\
\hline$\sigma_{x}$ & 0.225 & 0.227 & 0.240 & 0.263 & 0.132 & 0.103 \\
\hline$\beta$ & 2.682 & 2.460 & 2.553 & 2.346 & 4.956 & 6.420 \\
\hline$\alpha$ & 0.082 & 0.089 & 0.067 & 0.041 & 0.014 & 0.012 \\
\hline
\end{tabular}

score is $6 \%$ on all championships and of $5 \%$ on the 11 World Championships for which the ELO predictor is available. So, the accumulated conformance predictor is giving on the whole good results, on par with the ELO predictor. We will further discuss this predictor when we will compare the three predictors.

\subsection{Gain and distribution covariance}

The gain and distribution covariance section is partitioned into three subsections: correlation with the outcome of a game (4.3.1), conformance of play during a whole career (4.3.2), and predicting the results of World Championship matches (4.3.3).

\subsubsection{Correlation with the outcome of a game}

In this subsection we are going to see how computing the expected result of a game by using Ferreira's distribution method (presented in section 3.3) fares. Thus, for each game, I compute the vectors $R_{W}(\delta)$ and $R_{B}(\delta)$ of the distribution of $\delta$ for each player for the given game, and the convolution of the two distributions, which gives us the distribution of $R_{W-B}$. Then I compute the scalar product of this vector with the vector describing the expected gain, which is in Ferreira's paper $e=(0, \cdots, 0,0.5,1, \cdots, 1)$. The result should be the expected outcome of the given game.

The first goal here is thus to evaluate the correlation of this covariance indicator with the outcome of the games, as we did in subsection 4.2.1 for the accumulated conformance indicator. It can be done for raw $\delta$ (that is what Ferreira is doing in its paper), but it can also be extended to G\&B conformance and to ponderated "bi-linear" conformance. Results are available in Table 6 , where $\left(k_{1}=1.44, k_{2}=-3.53\right)$ are the values found in subsection 3.2.3 through linear regression and $\left(k_{1}=0.37, k_{2}=-3.70\right)$ are the optimal values found when optimizing the values of $k_{1}$ and $k_{2}$ with, here again, a Nelder-Mead simplex to get the best possible correlation.

The table also holds the mean $(\bar{x})$ of the estimated result (values are in $[-1,1])$, its standard deviation $\left(\sigma_{x}\right)$, and the values of $\beta$ and $\alpha$ which have been computed in exactly the same way as in the previous section. The mean of the actual game outcomes is 0.12 (56\% for White) and the standard deviation is 0.75 .

We can deduce a plethora of things from these results. First, while the mean is approximately correct (it is almost 0 , with a slight bias for White, as in the previous section), the standard deviation is much too small. This was not much of a concern regarding the accumulated conformance indicator in the previous section, which did not claim to represent the actual outcome of the game, but it is here a hint that something is not correct, as the interpretation of the scalar product of the covariance vector with the gain vector $e$ was supposed to be an estimation of the outcome of the games, and not to be only correlated with it. Thus, we have to apply a linear scaling function, with coefficients $\beta$ and $\alpha$ which 
are quite similar to the ones found for the accumulated conformance indicator in the previous section. Second, the best optimal correlation found ( 0.825 for ponderated conformance) is less than the best correlation found in section 4.2 for the optimal accumulated conformance indicator. We should have expected the opposite: the accumulated conformance indicator is a scalar value, and thus captures less information than this indicator, which "represents" a player's style by a vector instead of a scalar.

The first thing to do is to seriously reconsider the values of vector $e$. As a quick experiment, we set coefficients in $e$ according to the function:

$$
e(\delta)=0.5(1+\operatorname{th}(a \delta))
$$

Here, $\mathrm{a}$ is a suitable constant to determine. Using again a Nelder-Mead optimization but on three parameters $\left(k_{1}, k_{2}\right.$ and $a$ ), we find the optimal values $k_{1}=1.20, k_{2}=-3.41$ and $a=1.16$ with an optimal correlation of 0.875 , which is this time better than the one found for the best accumulated conformance indicator. In Fig. 9, we have the curve describing the shape of the coefficients of vector $e$.

In order to validate these coefficients, a second optimization was performed, on 21 variables, two for $k_{1}$ and $k_{2}$ and 19 for fitting 19 points of a spline. Coefficients were set to 0.5 at 0 and to 1.0 at +4 , and the 19 variables gave the value of the coefficient at $(0.2,0.4, \cdots, 3.6,3.8)$. The other values were interpolated. The shape of the curve is quite different, however the correlation is only slightly better (0.879), and the mean, the standard deviation, $\alpha$ and $\beta$ are similar ( $\beta$ is even higher). This means that, on the one hand, the correlation is not very sensitive to the parameters, and on the other hand that this indicator also needs to be "stretched" in order to predict the scores, just like the conformance indicator.

This is not really surprising. I have here mainly followed Ferreira's presentation and interpretation found in Ferreira (2012). In the paper, Ferreira links directly the distribution $R_{p 1-p 2}$ to the expected score of the game by the formula presented also here in section 3.3. This is however a little far-fetched. $R_{p 1-p 2}(x)$ is the probability that the score evolves by $x$ after a sequence of two moves: one white move followed by one black move. For example, if the score is $S$, then the probability that it remains $S$ after one white move followed by one black move is just $R_{p 1-p 2}(0)$, so $R_{p 1-p 2}$ is highly centered around 0 (after a pair of moves, the score does not change much). The distribution describing the evolution of the score after a sequence of 4 moves is the convolution of $R_{p 1-p 2}$ with itself, and the distribution

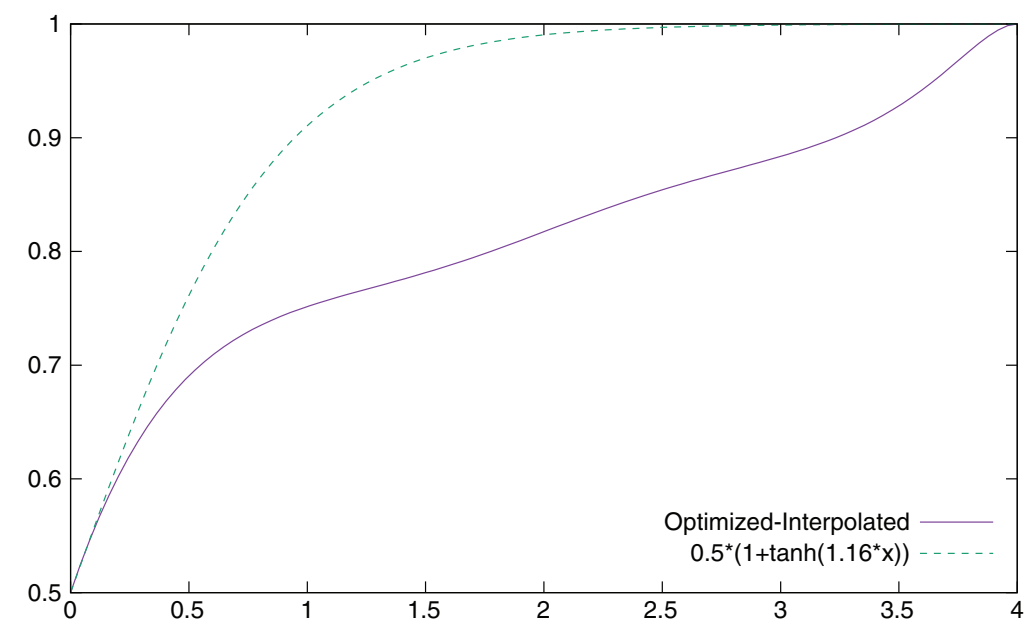

Fig. 9. Values of the coefficients of vector $e$ as a function of the difference of the two random variables. 


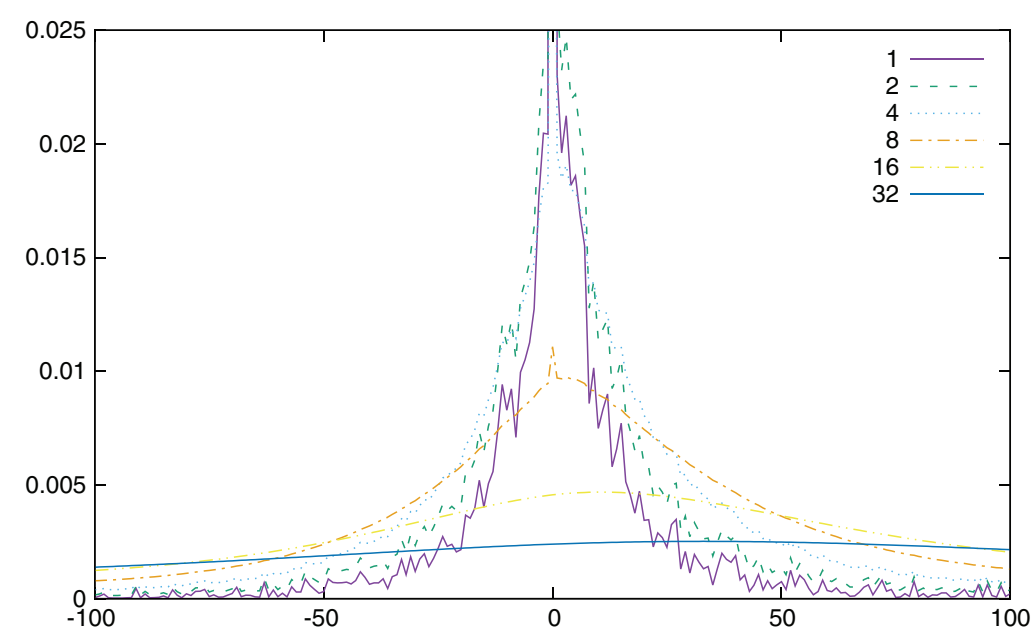

Fig. 10. Example of a distribution of $R_{w-b}$ from a Fischer-Spassky game, for 1, 2, 4, 8, 16 and 32 pairs of moves. The x-axis scale is in centipawns.

describing the evolution of the score after $2 n$ moves is $R_{p 1-p 2}^{n}$ (the convolution of $R$ by itself $n$ times).

For the sake of simplicity, we approximate in the next few lines $R$ by a normal distribution of parameters $\mu$ as mean and $\sigma$ as standard deviation (in Fig. 10 we have an example of the distribution of $R_{w-b}$; it is not normal, however when $n$ becomes larger, it takes the shape of a normal distribution, thanks to the central theorem limit). Then $R^{n}$ is a normal distribution of parameters $\mu_{n}=n \mu$ and $\sigma_{n}=\sqrt{n} \sigma$ : the distribution "shifts" to the right if $\mu$ is positive ( $p 1$ is the strongest player), and to the left if $\mu$ is negative ( $p 2$ is the strongest player), and it also "flattens", i.e., it is much less centered around $\mu_{n}$. If we consider that a victory is having a score $S>b$ after $n$ moves, then its probability is $\int_{b}^{+\infty} R^{n}=\left(1-\operatorname{erf}\left(\frac{b-n \mu}{\sqrt{2 n} \sigma}\right)\right) / 2$. Respectively, a draw would be $\int_{-b}^{+b} R^{n}=\left(\operatorname{erf}\left(\frac{b-n \mu}{\sqrt{2 n} \sigma}\right)+\operatorname{erf}\left(\frac{b+n \mu}{\sqrt{2 n} \sigma}\right)\right) / 2$ and a defeat $\int_{-\infty}^{-b} R^{n}=\left(1-\operatorname{erf}\left(\frac{b+n \mu}{\sqrt{2 n} \sigma}\right)\right) / 2$.

If we compute the limit when $n \rightarrow+\infty$ we see that all the density of the distribution goes to either side depending on the sign of $\mu$ : if player $p 1$ is the strongest (respectively weakest) $\mu$ is positive (respectively negative) and, at infinity, all games end in wins (respectively defeats) for White. Intuitively, the fundamental flaw in the hypothesis is that a chess game is finite and thus ends after the score reaches some given limit on either side, something we are not taking into account here, thus taking simply the limit is not correct either.

Instead of fitting the model to the data by using parameters $k_{1}, k_{2}$ and the elements of the gain vector, it is possible to compute the correlation of the estimated gain (here ${ }^{31} 0.5 \int_{-b}^{+b} R^{n_{0}}+1 \int_{b}^{+\infty} R^{n_{0}}$ ) with the actual result of the game as a function of $n_{0}$ and $b$. The results are displayed in Fig. 11, for $n_{0}=0, \cdots, 95$ and $b=0.0,0.2, \cdots 7.6,7.8$. They are excellent, with a maximal value for $\rho$ of $95 \%$, much higher than any other value we ever had.

There are many tuples $\left(n_{0}, b\right)$ for which the correlation is around $95 \%$.

\footnotetext{
${ }^{31}$ The value of the integrals can easily be computed from the actual discrete distributions by performing $n_{0}$ discrete convolutions.
} 


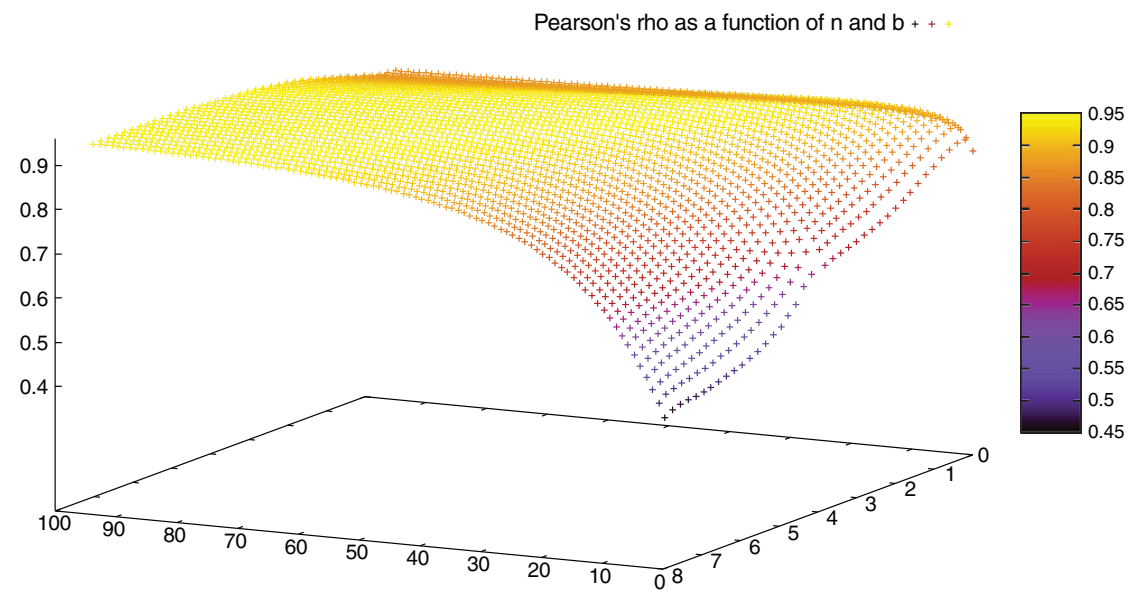

Fig. 11. $\rho$ as a function of $n_{0}$ (from 0 to 95$)$ and $b$ (from 0 to 8 ).
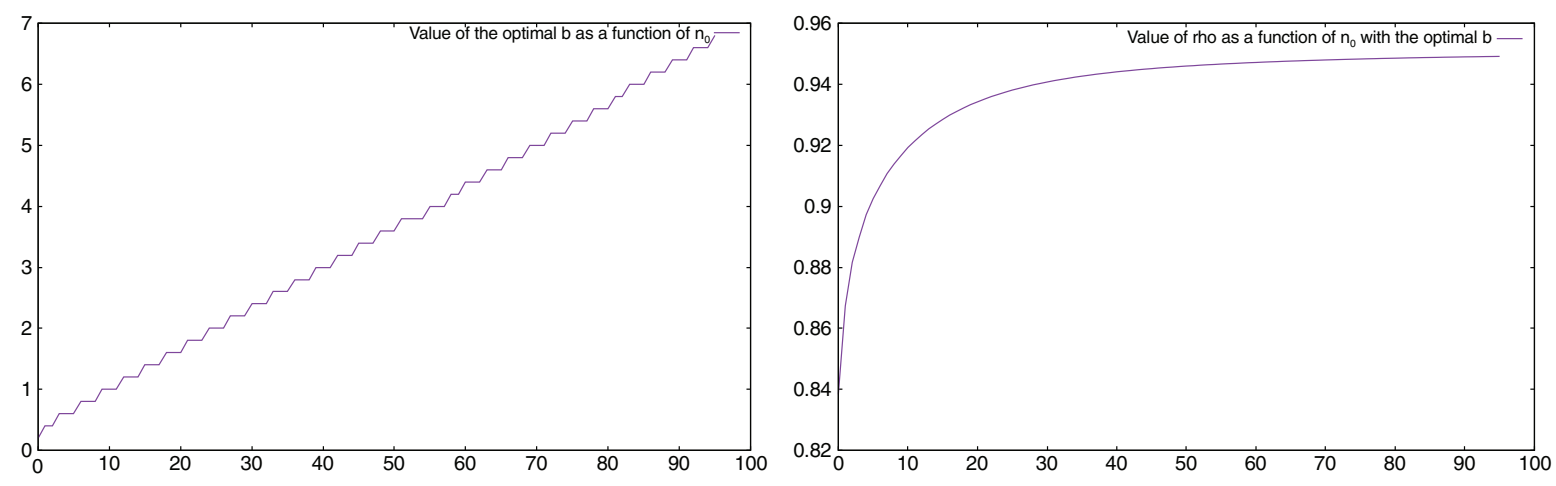

Fig. 12. Value of the optimal $b$ (left), and of $\rho$ with the optimal $b$ (right), as a function of $n_{0}$.

In the left part of Fig. 12 we display the optimal value of $b$ as a function of $n_{0}$. As predicted by the normal distribution approximation, $b$ grows almost linearly with $n_{0}$. The correlation is rising fast and $94 \%$ is reached for $n_{0}=29$ and $b=2.2$.

\subsubsection{Whole career}

The gain covariance representation is only able to provide results for head to head confrontations. It is not a scalar value and thus cannot be plot like the aggregated conformance indicator. However, as all results are available for all World Champions for all their active years, it is now possible to predict the outcome of a match between any World Champion from any active year with any other Champion taken in any active year; it is even possible to predict the result of Fischer 1970 against Fischer 1971.

A first experiment was done using the most basic settings, i.e., setting $n_{0}$ to 0 (which is exactly Ferreira's interpretation). This "Battle Royale" which consisted in predicting the result of around 300,000 possible match combinations, was performed in a few minutes by the computer. The result is a 14 megabytes database which gives the predicted outcome of the games between any two World Champions for any year.

Now, for each player, the "best year" was found by searching for the year where the player had the largest number of victories against all other players and all other years. The results were as follows: 
Table 7

Head to head match result predictions between different World Champions in their best year using the Covariance indicator with $n_{0}=0$

\begin{tabular}{lcccccccccccccccccccc}
\hline & $\mathrm{Ca}$ & $\mathrm{An}$ & $\mathrm{Kr}$ & $\mathrm{Ka}$ & $\mathrm{To}$ & $\mathrm{Fi}$ & $\mathrm{Kh}$ & $\mathrm{Po}$ & $\mathrm{Bo}$ & $\mathrm{Ka}$ & $\mathrm{Ka}$ & $\mathrm{Ca}$ & $\mathrm{La}$ & $\mathrm{Pe}$ & $\mathrm{Ta}$ & $\mathrm{Sm}$ & $\mathrm{Eu}$ & $\mathrm{Al}$ & $\mathrm{Sp}$ & $\mathrm{St}$ \\
\hline Carlsen & & 50 & 50 & 51 & 51 & 52 & 52 & 52 & 52 & 53 & 53 & 53 & 53 & 54 & 54 & 54 & 54 & 54 & 54 & 55 \\
Anand & 50 & & 50 & 50 & 51 & 51 & 51 & 52 & 52 & 52 & 52 & 53 & 53 & 53 & 53 & 53 & 53 & 54 & 54 & 55 \\
Kramnik & 50 & 50 & & 50 & 51 & 51 & 51 & 52 & 52 & 52 & 52 & 53 & 53 & 53 & 53 & 53 & 53 & 53 & 54 & 55 \\
Kasparov & 49 & 50 & 50 & & 50 & 51 & 51 & 51 & 52 & 52 & 52 & 52 & 53 & 53 & 53 & 53 & 53 & 53 & 53 & 55 \\
Topalov & 49 & 49 & 49 & 50 & & 50 & 51 & 51 & 51 & 51 & 52 & 52 & 52 & 52 & 52 & 53 & 53 & 53 & 53 & 54 \\
Fischer & 48 & 49 & 49 & 49 & 50 & & 50 & 50 & 51 & 51 & 51 & 51 & 52 & 52 & 52 & 52 & 52 & 52 & 53 & 54 \\
Khalifman & 48 & 49 & 49 & 49 & 49 & 50 & & 50 & 51 & 51 & 51 & 51 & 52 & 52 & 52 & 52 & 52 & 52 & 53 & 54 \\
Ponomariov & 48 & 48 & 48 & 49 & 49 & 50 & 50 & & 50 & 50 & 51 & 51 & 51 & 51 & 51 & 52 & 52 & 52 & 52 & 53 \\
Botvinnik & 48 & 48 & 48 & 48 & 49 & 49 & 49 & 50 & & 50 & 50 & 51 & 51 & 51 & 51 & 51 & 51 & 52 & 52 & 53 \\
Kasimdzhanov & 47 & 48 & 48 & 48 & 49 & 49 & 49 & 50 & 50 & & 50 & 51 & 51 & 51 & 51 & 51 & 51 & 51 & 52 & 53 \\
Karpov & 47 & 48 & 48 & 48 & 48 & 49 & 49 & 49 & 50 & 50 & & 50 & 51 & 51 & 51 & 51 & 51 & 51 & 52 & 53 \\
Capablanca & 47 & 47 & 47 & 48 & 48 & 49 & 49 & 49 & 49 & 49 & 50 & & 50 & 50 & 51 & 51 & 51 & 51 & 51 & 53 \\
Lasker & 47 & 47 & 47 & 47 & 48 & 48 & 48 & 49 & 49 & 49 & 49 & 50 & & 50 & 50 & 50 & 50 & 51 & 51 & 52 \\
Petrosian & 46 & 47 & 47 & 47 & 48 & 48 & 48 & 49 & 49 & 49 & 49 & 50 & 50 & & 50 & 50 & 50 & 51 & 51 & 52 \\
Tal & 46 & 47 & 47 & 47 & 48 & 48 & 48 & 49 & 49 & 49 & 49 & 49 & 50 & 50 & & 50 & 50 & 50 & 51 & 52 \\
Smyslov & 46 & 47 & 47 & 47 & 47 & 48 & 48 & 48 & 49 & 49 & 49 & 49 & 50 & 50 & 50 & & 50 & 50 & 51 & 52 \\
Euwe & 46 & 47 & 47 & 47 & 47 & 48 & 48 & 48 & 49 & 49 & 49 & 49 & 50 & 50 & 50 & 50 & & 50 & 51 & 52 \\
Alekhine & 46 & 46 & 47 & 47 & 47 & 48 & 48 & 48 & 48 & 49 & 49 & 49 & 49 & 49 & 50 & 50 & 50 & & 50 & 52 \\
Spassky & 46 & 46 & 46 & 47 & 47 & 47 & 47 & 48 & 48 & 48 & 48 & 49 & 49 & 49 & 49 & 49 & 49 & 50 & & 51 \\
Steinitz & 45 & 45 & 45 & 45 & 46 & 46 & 46 & 47 & 47 & 47 & 47 & 47 & 48 & 48 & 48 & 48 & 48 & 48 & 49 & \\
\hline
\end{tabular}

Alekhine (1921), Anand (2010), Botvinnik (1945), Capablanca (1918), Carlsen (2013), Euwe (1934), Fischer (1972), Karpov (1988), Kasimdzhanov (2013), Kasparov (2000), Khalifman (2013), Kramnik (2007), Lasker (1907), Petrosian (1962), Ponomariov (2013), Smyslov (1964), Spassky (1965), Steinitz (1872), Tal (1981), Topalov (2006).

Some results might seem surprising. For example, it is usually supposed that Botvinnik had been playing at his peak when he was World Champion (from 1948 to 1963). However, when looking carefully, 1945 was an exceptional year for him: he won the USSR Championship with an amazing 15 out of 17 possible points, at a time when there were almost no international competitions, and where the USSR Championship was probably the strongest possible competition. So it is quite possible that 1945 is indeed the year he played at his best. A second quite surprising results is Tal's best years. But there again Tal reached his peak ELO rating in 1980, far from the years he was World Champion.

Then we extracted from the database the results of the head to head predictions for these players taken this particular year. The results are displayed in Table 7 . The results are not exactly symmetric as playing as White and playing as Black give different results as explained above.

A second similar experiment was done, with different parameters. Here $n_{0}=32$ and $b=2.4$, which is supposed to yield "better" results. These results are somewhat different from the previous ones. The best years are: Alekhine (1931), Anand (2011), Botvinnik (1945), Capablanca (1924), Carlsen (2013), Euwe (1934), Fischer (1971), Karpov (1977), Kasimdzhanov (2013), Kasparov (2001), Khalifman (2013), Kramnik (2000), Lasker (1907), Petrosian (1962), Ponomariov (2013), Smyslov (1964), Spassky (1970), Steinitz (1873), Tal (1967), Topalov (2005). The results are presented in Table 8. We see that Fischer fell to almost the end of the ranking, while Capablanca almost reached the top. 
Table 8

Head to head match result predictions between different World Champions in their best year using the Covariance indicator with $n_{0}=32$ and $b=2.4$

\begin{tabular}{|c|c|c|c|c|c|c|c|c|c|c|c|c|c|c|c|c|c|c|c|c|}
\hline & $\mathrm{Ka}$ & $\mathrm{Kr}$ & $\mathrm{Ca}$ & $\mathrm{Ca}$ & $\mathrm{Ka}$ & An & $\mathrm{Pe}$ & $\mathrm{Kh}$ & $\mathrm{Ka}$ & $\mathrm{Sm}$ & $\mathrm{La}$ & Bo & $\mathrm{Eu}$ & To & $\mathrm{Ta}$ & Po & $\mathrm{Al}$ & $\mathrm{Sp}$ & $\mathrm{Fi}$ & St \\
\hline Kasparov & & 51 & 51 & 52 & 52 & 53 & 53 & 53 & 53 & 54 & 55 & 55 & 57 & 58 & 60 & 60 & 61 & 64 & 66 & 69 \\
\hline Kramnik & 49 & & 50 & 51 & 51 & 52 & 52 & 53 & 53 & 53 & 54 & 54 & 57 & 57 & 59 & 60 & 60 & 64 & 65 & 68 \\
\hline Carlsen & 49 & 50 & & 51 & 51 & 52 & 52 & 53 & 53 & 53 & 54 & 54 & 57 & 57 & 60 & 60 & 60 & 64 & 65 & 69 \\
\hline Capablanca & 48 & 49 & 49 & & 50 & 51 & 51 & 51 & 52 & 52 & 53 & 53 & 55 & 56 & 58 & 58 & 58 & 62 & 63 & 66 \\
\hline Karpov & 48 & 49 & 49 & 50 & & 51 & 51 & 51 & 51 & 52 & 52 & 53 & 55 & 55 & 58 & 58 & 58 & 62 & 63 & 66 \\
\hline Anand & 47 & 48 & 48 & 49 & 49 & & 50 & 51 & 51 & 51 & 52 & 52 & 55 & 55 & 58 & 58 & 58 & 62 & 63 & 66 \\
\hline Petrosian & 47 & 48 & 48 & 49 & 49 & 50 & & 50 & 51 & 51 & 52 & 52 & 54 & 55 & 57 & 57 & 58 & 61 & 63 & 65 \\
\hline Khalifman & 47 & 47 & 48 & 49 & 49 & 50 & 50 & & 50 & 51 & 51 & 52 & 54 & 54 & 57 & 57 & 57 & 61 & 62 & 65 \\
\hline Kasimdzhanov & 47 & 48 & 48 & 49 & 49 & 50 & 50 & 50 & & 51 & 51 & 52 & 54 & 54 & 57 & 57 & 57 & 61 & 62 & 65 \\
\hline Smyslov & 46 & 47 & 47 & 48 & 48 & 49 & 49 & 49 & 49 & & 50 & 51 & 53 & 53 & 56 & 56 & 56 & 60 & 61 & 64 \\
\hline Lasker & 46 & 46 & 46 & 48 & 48 & 48 & 48 & 49 & 49 & 50 & & 50 & 53 & 53 & 55 & 56 & 56 & 60 & 61 & 63 \\
\hline Botvinnik & 45 & 46 & 46 & 47 & 47 & 48 & 48 & 48 & 49 & 49 & 50 & & 52 & 53 & 55 & 55 & 56 & 59 & 61 & 63 \\
\hline Euwe & 43 & 44 & 43 & 45 & 45 & 45 & 46 & 46 & 46 & 47 & 47 & 48 & & 50 & 53 & 53 & 53 & 57 & 58 & 61 \\
\hline Topalov & 42 & 43 & 43 & 45 & 45 & 45 & 45 & 46 & 46 & 47 & 47 & 47 & 50 & & 52 & 53 & 53 & 57 & 58 & 61 \\
\hline Tal & 40 & 41 & 40 & 42 & 43 & 43 & 43 & 43 & 44 & 45 & 45 & 45 & 47 & 48 & & 50 & 51 & 55 & 55 & 58 \\
\hline Ponomariov & 40 & 41 & 40 & 42 & 42 & 43 & 43 & 43 & 43 & 44 & 45 & 45 & 47 & 48 & 50 & & 51 & 54 & 55 & 59 \\
\hline Alekhine & 40 & 40 & 40 & 42 & 42 & 42 & 43 & 43 & 43 & 44 & 44 & 44 & 47 & 47 & 49 & 50 & & 54 & 55 & 58 \\
\hline Spassky & 36 & 36 & 36 & 38 & 38 & 38 & 39 & 39 & 39 & 40 & 40 & 41 & 43 & 43 & 46 & 46 & 46 & & 51 & 54 \\
\hline Fischer & 34 & 35 & 35 & 37 & 37 & 37 & 38 & 38 & 38 & 39 & 39 & 40 & 42 & 42 & 45 & 45 & 45 & 49 & & 53 \\
\hline Steinitz & 31 & 32 & 31 & 34 & 34 & 34 & 35 & 35 & 35 & 36 & 37 & 37 & 39 & 39 & 42 & 42 & 43 & 46 & 47 & \\
\hline
\end{tabular}

There are some common factors in both rankings: according to the conformance indicator, the level of chess has been increasing through the years. There are more "contemporary" players in the top of this ranking than players from the previous generations. There are also important differences; this is probably telling us that this indicator is somewhat "flawed" for the same reasons as the aggregated conformance indicator: it does not take into account the "context" of the move: making a small mistake when the game is already lost or won has not the same significance as making it when the game issue is not decided yet, while this indicator is "averaging" them. A longer study is necessary to assess exactly why some players are more "unstable" than others. However the example of Fischer is somewhat significant: let us remember that, with the aggregated conformance indicator, Fischer was also "topping" the rankings regarding his ability to find the exact "best" move, but was much more "ordinary" when considering aggregated conformance for $\delta \leq 0.3$. This might mean that he was playing perfectly very often but could also make "larger" mistakes more often than some other players. The question again is: under what circumstances was he making such mistakes? This problem is exactly what I expect to correct with the Markovian predictor.

\subsubsection{Predicting the results of World Championships}

Below, we compare the score predicted for World Championships by the Covariance predictor to (1) the actual score and to (2) the score predicted using ELO tables as we did for the accumulated conformance indicator in subsection 4.2.4. The results are also presented in Table 5 in column Cov $_{s}$. The predictor is computed using $n_{0}=32$ and $b=2.4$.

The mean difference between the actual score and the accumulated conformance predicted score is $8 \%$ on all championships and of $9 \%$ on the 11 World Championships for which the ELO predictor is 
available. So, in a quite paradoxical way, the covariance predictor is less efficient than the accumulated conformance predictor, even if it is better correlated to the result of individual games.

\subsection{The Markovian predictor}

The Markovian predictor presented in section 3.4 relies on transition matrices which represent for each value of the evaluation function the probability of the value of the evaluation function in the next step. This solves the problem presented in the previous sections regarding the "context" of a mistake. However, to operate properly, the Markovian predictor requires a large amount of data to build matrices which are statistically significant. Thus it is not possible to use it and/or validate it on a single game, because there are simply not sufficient data. The Markovian predictor is designed to evaluate a player on a collection of games and not on single games, which is quite different from the previous two. In the next three subsections we explain first how to compute efficiently transition matrices (4.4.1), then we compute the Markovian predictor on whole careers (4.4.2) and finally we use it to compute predictions for World Championships matches (4.4.3).

\subsubsection{Computing efficiently transition matrices}

There are two antagonist objectives when building transition matrices. On the one hand, the more classes (rows) we have, and the better is the modeling of the stochastic process. On the other hand, it is important to have "sufficient" moves played in each class (row of the matrix), in order to have a significant statistical estimation of all the parameters of this class.

As matrices are computed for each year the player was active, it is mandatory to set a lower bound to the number of moves played during one year to declare the player "active". This is not as simple as it seems. Some players (such as Botvinnik for example) used to play a low number of games between championships. Some retired for long periods (Fischer retired for 18 months from mid 68 to mid 70). After examining the careers of different players, the lower bound for the number of moves played was set to 500, which seems to make a proper distinction between years of activity and years of semi-retirement (Fischer played some demonstration games in 1969 that cannot be considered as significant).

The second parameter to choose is the value of the upper and lower bounds $b_{\text {inf }}$ and $b_{\text {sup }}$. There again, the larger the value, the better the prediction of the process should be. However, here again, it is important to have sufficient positions when the evaluation of the current game is below $b_{\text {inf }}$ and above $b_{\text {sup }}$. A statistical analysis of the games of the players considered shows that it is difficult to find many moves played below $b_{\text {inf }}$ if $b_{\text {inf }}$ is too large. There are two main reasons: on the one hand, world class players usually do not lose their games; on the other hand, when they are in this kind of situation, they seem to resign pretty soon, which reduces the number of moves available. The same goes on a lesser extent for $b_{\text {sup }}$; even if their opponents are less strong, they usually resign pretty fast when the position becomes bad against a world class player. The side effect of choosing $b_{\text {sup }}$ too low (respectively $b_{\text {inf }}$ too high), is that the expected percentage of won (respectively lost) games will be higher, while the expected percentage of drawn games will be lower.

The underlying interpretation of the stationary vector is that the last component of the stationary vector represents games won and that the expected gain should be 1 for this class ${ }^{32}$, that the "middle" elements correspond to draw with unbalanced material, while the first component represent games lost. We are thus using $e=(0,0.5 \cdots, 0.5,1)$ as the gain vector, and we compute the

\footnotetext{
${ }^{32}$ This is of course not true as we will see later: around only $90 \%$ of games are won when a player has once a position better than 1.8 , not $100 \%$.
} 
expected output of the game by making the scalar product of the stationary vector $\pi$ with the gain vector $e$.

Let us notice first that we cannot use, to compute the values of the $e$ vector, the same optimization method as in the previous section for the covariance gain vector. The optimization in the previous section can be performed because we can compute for each game the expected result, compare it to the actual result and perform a least squares method to reduce the discrepancy. The Markovian method works only on a large set of games, because it requires a large amount of data. It is impossible to compute one matrix for one game. We could have performed an optimization by computing the matrix on a large number of games, and then compare the expected average computed outcome with the actual one. However, we decided not to perform this optimization step, for different reasons.

- On the one hand, the Markovian process and the computation of the stationary vector takes into account the idea that a high value (low value) leads usually the game to a higher value (respectively lower value) and ultimately to one of the extremal class. Thus the probabilities represented in the stationary vector by the "not extremal" classes are really the probabilities of not going to one of the extremal class when $t \rightarrow+\infty$, and thus they represent a draw with more or less unbalanced material.

- On the other hand, the gain expectancy associated to a class cannot be estimated in an intrinsic way: it depends, not only on the player, but also on his opponents, as we can only estimate it from the player's games. Moreover, in a game between two players, what vector do we choose: player one's vector, player two's vector or an average of the two? To solve this last problem we could try to find a general "gain expectancy" vector, either by trying to make a least square regression on actual data, or by deducing it from many games played by the computer in autoplay. But using this interpretation would defeat the very idea that different human players have different capacities, and should thus have different "gain vectors" if we choose to interpret them that way.

- Last, but not least, not performing any "fitting" of the model to the data guarantees that the Markovian model remains, in a way, "pure", as it is completely independent of the players on which the study is performed. The only thing that depends on the player is its Markovian matrix.

This choice will be discussed in subsection 4.4.3, when I will compare the "pure" Markovian model to the other predictors regarding its capacity to predict the outcome of a set of games.

It is possible to try to have an idea of the estimation of the induced error. In Fig. 13, we see for some selected players the expected value of the result as a function of the evaluation of the best position reached by the player during the game (the statistics are computed on all games). For example, if we consider Emmanuel Lasker, he won on the average 0.65 points when, during a game, he reached at least once a position valued 0 . He won 0.85 points when he reached at least once a position valued 1.00 (100cp), 0.94 points for $2.00,0.96$ points for $3.00^{33}$. So we make an approximation of 0.06 points when setting $b_{\text {sup }}$ to 2.00 , and an error of 0.04 points when setting it to 3.00 .

These values are approximately the same for all the World Champions considered in this study, except for Wilhelm Steinitz, who is clearly below. We also notice that they are well over the "All Players"

\footnotetext{
${ }^{33}$ Intuitively, this curve represents the capacity of a player to "grab opportunities" and to "win" a game as soon as a "good position" is reached. However it is important to remember that it depends on the opponents of a given player during his career (and also on the engine doing the evaluation, but this induces only a shifting of the curve). So this estimation is in no way "intrinsic".
} 


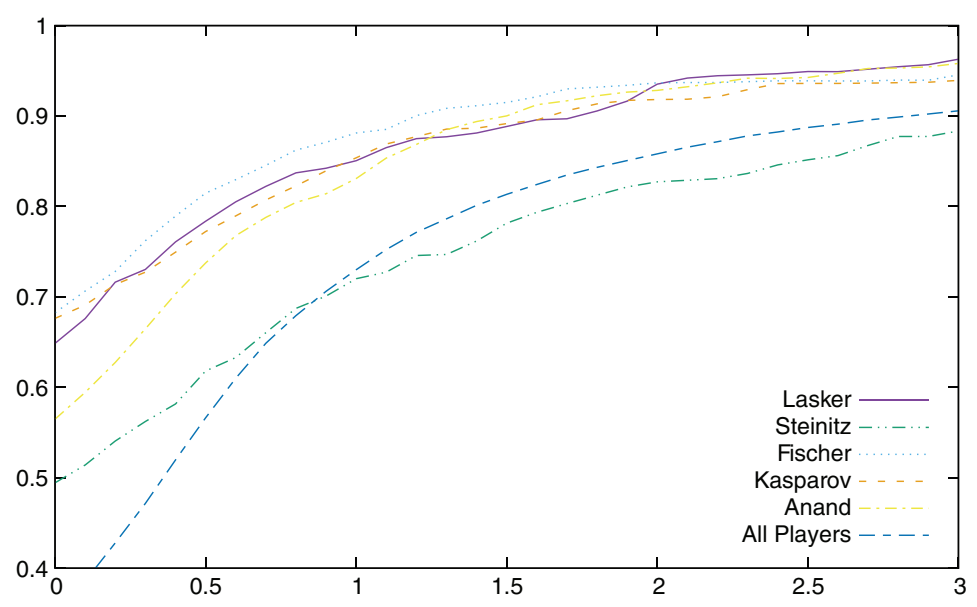

Fig. 13. Expected value of gain as a function of the highest evaluation reached in the game.

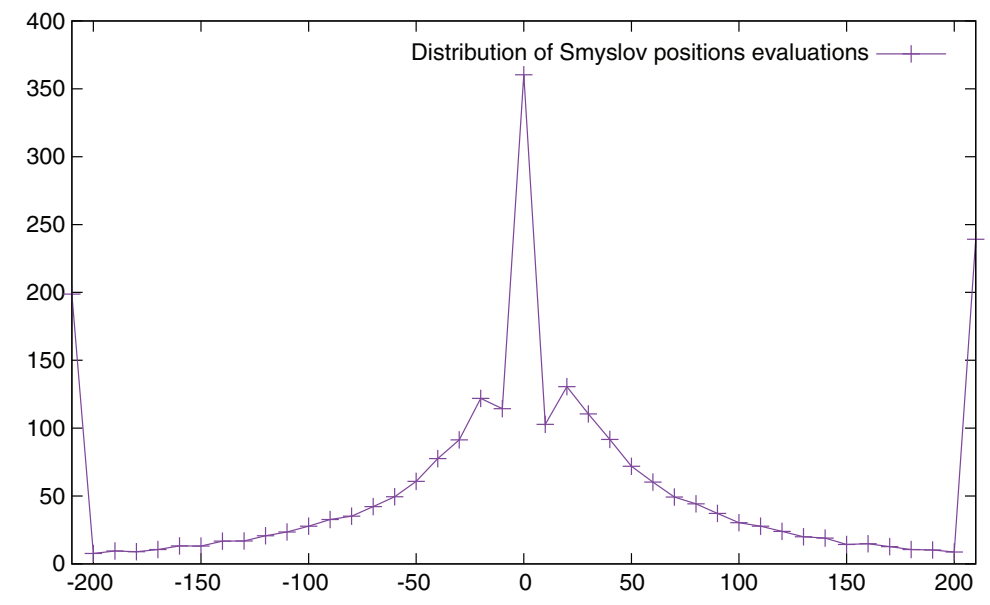

Fig. 14. Average number of positions by year as a function of the evaluation of the positions.

curve $^{34}$. The difference between 2.0 and 3.0 is small and thus a reasonable choice seems to be +2 as $b_{\text {sup }}$. The same study with quite identical results can be done for $b_{\text {inf }} \cdot+2 /-2$ is also the value chosen as the limit of won/lost games by Guid and Bratko in their study, and these values give us sufficient moves in the extremal classes to have statistical consistent samples.

The last value to choose is the grain $g$, which is the size of each class, and which thus sets also the number of classes (rows and columns of the matrices). The statistical analysis of position evaluations showed that choosing a single $g$ was a poor idea. Moves made are usually made when the evaluation is close to 0 , and their distribution is "Gaussian".

Fig. 14 represents the distribution of the evaluation of the positions of Vassily Smyslov during his extremely long and competitive career. $b_{\text {inf }}$ and $b_{\text {sup }}$ were set to $-2 /+2$, and $g$ was set to 10 centipawns. -210 represents all positions with an evaluation of -200 or below, -200 the positions with an evaluation between -200 and -190 and so on. There are 151,489 positions over 60 years, or an average of almost 2500 positions by year ( 5 times the limit of 500 moves).

\footnotetext{
${ }^{34}$ The "All Players" curve is the statistics for all players in the study, which include all World Champions and all the opponents they played against.
} 


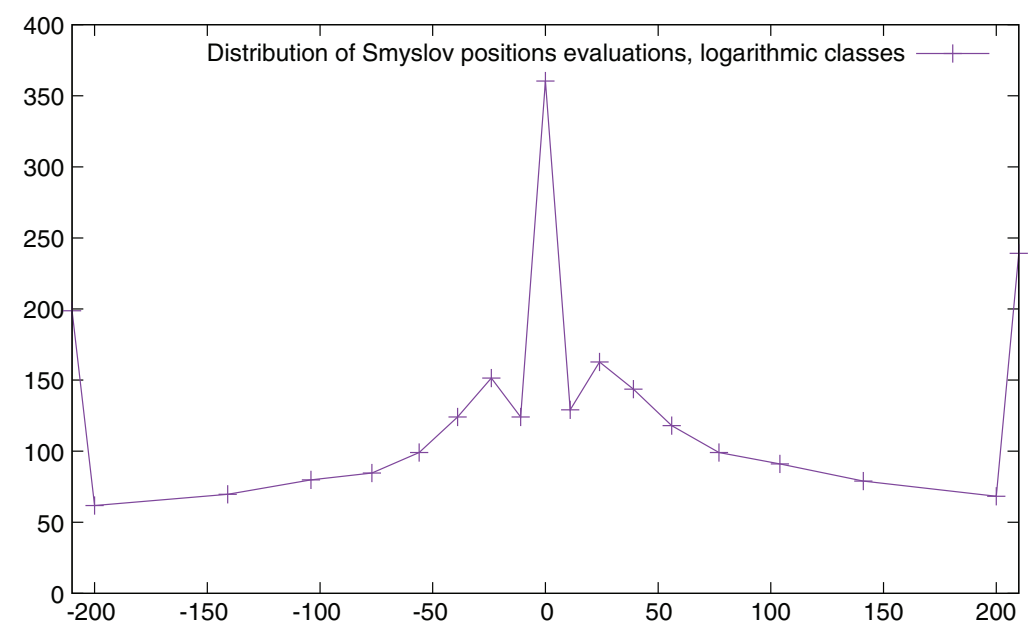

Fig. 15. Average number of positions by year as a function of the evaluation of the positions, using logarithmic size classes.

The positions evaluated as 0 are a class of their own as it is the positions which are draws, and there are lots of them. This is understandable as players often keep on playing in some positions that computer programs, especially with endgame databases, identify early as draws. Some classes are ridiculously small; for example the 200 class contains only 8 positions/year, and this is only on the average. A full statistical analysis for all players and all years demonstrated that with this distribution, there were some classes which were empty during "active" years.

Thus the number of classes was reduced to 19, and the width of each class was computed in order to better balance the number of elements by class. As the distribution looked Gaussian, the size of classes were set to follow a logarithmic function. The three special classes (above $b_{\text {sup }}$, below $b_{\text {inf }}$ and 0 ) are kept unchanged, while a scaling factor is applied to the size of classes equal to:

$$
f=\frac{e^{-v / 100}-e^{-b_{\text {sup }} / 100}}{e^{0}-e^{-b_{\text {sup }} / 100}} \text { for } v>0
$$

A similar symmetric factor was applied for $v<0$. The results are presented in Fig. 15. The central class (positions evaluated to 0 ) and the classes representing positions over $b_{\text {sup }}$ or below $b_{\text {inf }}$ are classes of their own. The classes closest to zero on either side are 10cp wide, and the width of classes grows as we get further from 0 , with the last classes being $50 \mathrm{cp}$ wide. This new distribution gives classes with a minimal number of around 50 elements, which is a significant sample.

A last improvement was made to the system. In order to stabilize the matrices, and to prevent jumps from year to year, the positions of the previous years are taken into account, but with an exponential forgetting factor of 2 (positions of the previous year count as half, position of $n-2$ count as one quarter, etc.). It would have probably been better to use a sliding time window, but unfortunately dates in the database are often reduced to the year and do not mention the month.

It is now possible to compute the transition matrices, which are square 19x19 matrices. Taking again as an example Fischer and Spassky in 1971, the new stationary vector is now:

$$
\begin{aligned}
v= & (0.10,0.02,0.01,0.01,0.02,0.02,0.02,0.02,0.02 \\
& 0.11,0.03,0.03,0.04,0.04,0.04,0.03,0.02,0.07,0.36)
\end{aligned}
$$



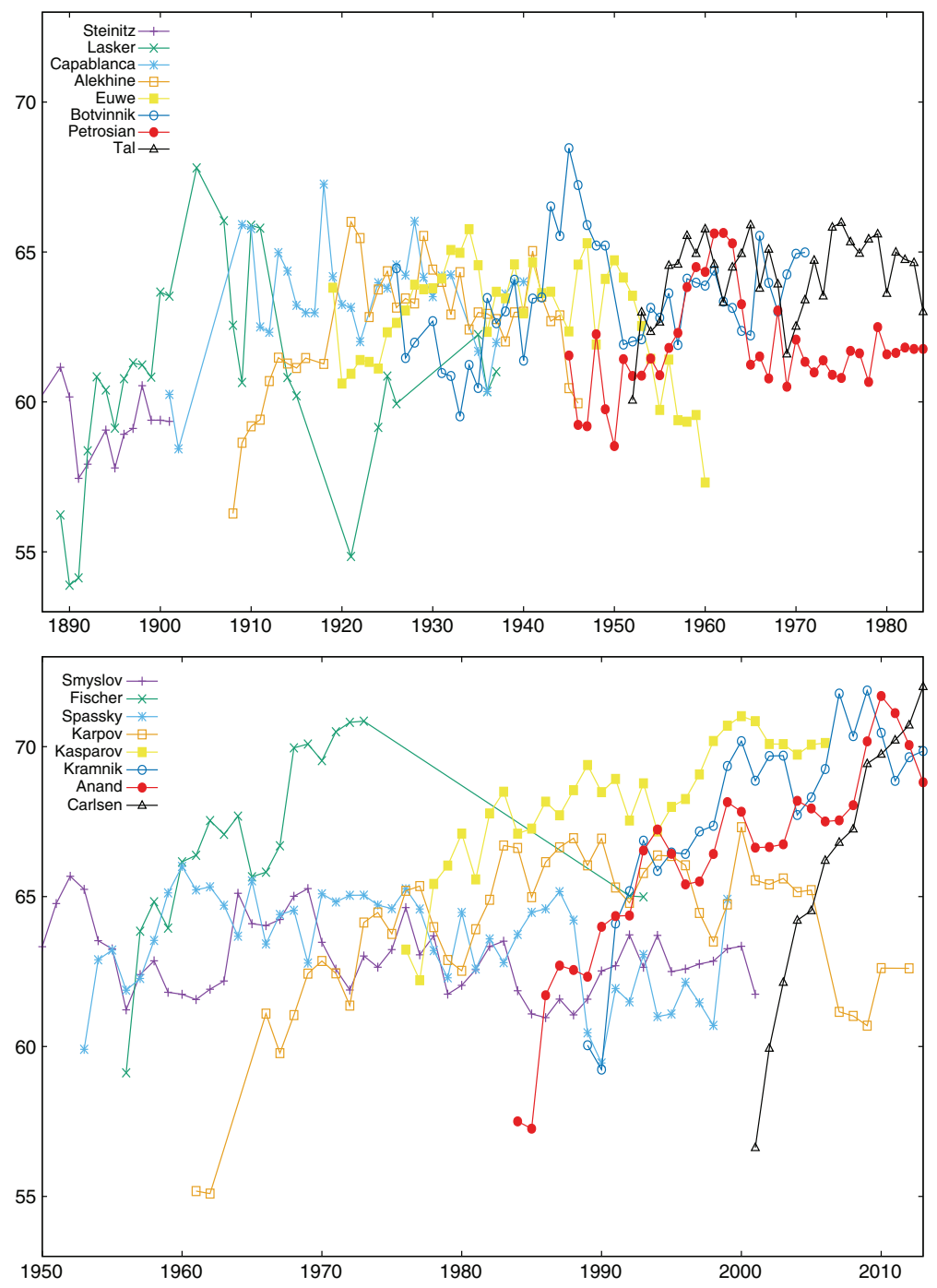

Fig. 16. Strength at $0 \mathrm{cp}$.

This represents a $36 \%$ win for Fischer and a $10 \%$ win for Spassky ${ }^{35}$.

A last important comment: as with any statistical methods, data are aggregated here solely based on some specific criteria (the value of the evaluation function), disregarding all other parameters. For example, the material still present on the board is not taken into account, while it seems pretty clear that the variations in the evaluation function are not of the same nature at the beginning of a game and at the end of a game. It would be interesting to try to create more complex classes, using the material present on the board as a second criteria. This is probably difficult to do; even if only three main subclasses are used (opening, mid-game and ending), this would subdivide each class into three classes, and the problem of having sufficient samples would arise again.

\footnotetext{
${ }^{35}$ The example was really chosen at random.
} 

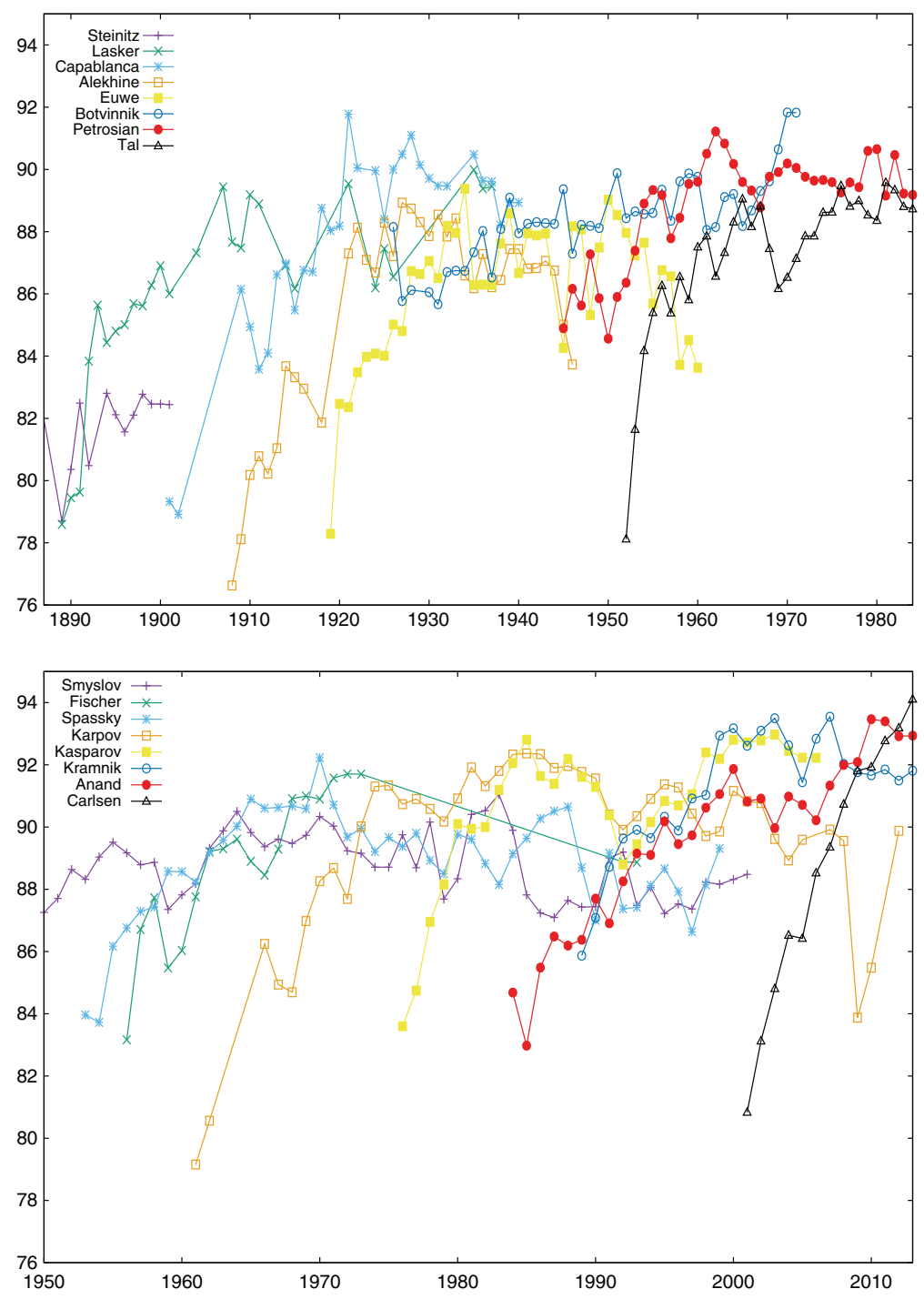

Fig. 17. Strength at 0-30cp.

\subsubsection{Whole career}

As in subsection 4.3.2, a "Battle Royale" was performed, and for each player, the "best year" was found by searching for the year where the player had the largest number of victories against all other players and all other years. The results were as follows: Carlsen: 2013, Kramnik: 1999, Fischer: 1971, Kasparov: 2001, Anand: 2008, Khalifman: 2010, Smyslov: 1983, Petrosian: 1962, Karpov: 1988, Kasimdzhanov: 2011, Botvinnik: 1945, Ponomariov: 2011, Lasker: 1907, Spassky: 1970, Topalov: 2008, Capablanca: 1928, Euwe: 1941, Tal: 1981, Alekhine: 1922, Steinitz: 1894.

The results are displayed in Table 9. Here again, the results are not exactly symmetric as playing as White and playing as Black give different results as explained above. It is not straightforward to deduce an absolute ranking from it; for example, Tal is performing consistently better than Euwe against stronger players, but is losing to Euwe (with a small margin). 
Table 9

Head to head match result predictions between different World Champions in their best year

\begin{tabular}{lccccccccccccccccccccc}
\hline & $\mathrm{Ca}$ & $\mathrm{Kr}$ & $\mathrm{Fi}$ & $\mathrm{Ka}$ & $\mathrm{An}$ & $\mathrm{Kh}$ & $\mathrm{Sm}$ & $\mathrm{Pe}$ & $\mathrm{Kp}$ & $\mathrm{Ks}$ & $\mathrm{Bo}$ & $\mathrm{Po}$ & $\mathrm{La}$ & $\mathrm{Sp}$ & $\mathrm{To}$ & $\mathrm{Ca}$ & $\mathrm{Ta}$ & $\mathrm{Eu}$ & $\mathrm{Al}$ & $\mathrm{St}$ \\
\hline Carlsen & & 52 & 54 & 54 & 57 & 58 & 57 & 58 & 56 & 60 & 61 & 59 & 60 & 61 & 61 & 64 & 66 & 69 & 70 & 82 \\
Kramnik & 49 & & 52 & 52 & 55 & 56 & 56 & 57 & 55 & 59 & 60 & 58 & 60 & 60 & 60 & 63 & 65 & 68 & 70 & 83 \\
Fischer & 47 & 49 & & 51 & 53 & 57 & 56 & 57 & 56 & 59 & 60 & 60 & 61 & 61 & 62 & 64 & 68 & 70 & 73 & 85 \\
Kasparov & 47 & 49 & 50 & & 53 & 54 & 54 & 54 & 53 & 57 & 58 & 56 & 56 & 58 & 58 & 60 & 62 & 66 & 68 & 82 \\
Anand & 44 & 46 & 48 & 48 & & 54 & 52 & 53 & 53 & 57 & 56 & 57 & 57 & 59 & 59 & 62 & 64 & 69 & 71 & 86 \\
Khalifman & 43 & 45 & 44 & 47 & 47 & & 50 & 51 & 52 & 53 & 54 & 55 & 55 & 56 & 56 & 60 & 62 & 64 & 67 & 79 \\
Smyslov & 43 & 45 & 45 & 47 & 49 & 51 & & 50 & 51 & 53 & 55 & 54 & 54 & 54 & 55 & 59 & 63 & 64 & 68 & 82 \\
Petrosian & 43 & 44 & 45 & 47 & 49 & 50 & 51 & & 52 & 53 & 54 & 54 & 55 & 55 & 56 & 59 & 63 & 63 & 67 & 80 \\
Karpov & 44 & 46 & 45 & 48 & 48 & 49 & 50 & 49 & & 51 & 52 & 52 & 52 & 52 & 52 & 56 & 58 & 60 & 63 & 76 \\
Kasimdzhanov & 41 & 43 & 42 & 45 & 45 & 48 & 48 & 48 & 50 & & 52 & 52 & 52 & 54 & 53 & 56 & 60 & 62 & 65 & 80 \\
Botvinnik & 40 & 41 & 41 & 44 & 45 & 48 & 46 & 48 & 49 & 49 & & 50 & 54 & 52 & 52 & 56 & 60 & 60 & 64 & 80 \\
Ponomariov & 42 & 43 & 41 & 45 & 44 & 47 & 47 & 47 & 49 & 49 & 51 & & 51 & 52 & 52 & 55 & 58 & 59 & 62 & 77 \\
Lasker & 41 & 41 & 40 & 45 & 44 & 46 & 47 & 46 & 49 & 49 & 48 & 50 & & 51 & 50 & 54 & 58 & 59 & 63 & 78 \\
Spassky & 40 & 41 & 40 & 43 & 42 & 45 & 47 & 46 & 48 & 47 & 49 & 49 & 50 & & 51 & 53 & 58 & 57 & 61 & 75 \\
Topalov & 40 & 41 & 39 & 44 & 42 & 45 & 46 & 45 & 49 & 48 & 49 & 49 & 50 & 51 & & 54 & 57 & 57 & 61 & 75 \\
Capablanca & 37 & 38 & 37 & 41 & 39 & 42 & 42 & 42 & 45 & 45 & 45 & 47 & 47 & 48 & 47 & & 53 & 54 & 59 & 76 \\
Tal & 35 & 36 & 34 & 39 & 37 & 39 & 39 & 38 & 43 & 41 & 41 & 43 & 43 & 43 & 44 & 48 & & 49 & 54 & 72 \\
Euwe & 32 & 33 & 32 & 36 & 32 & 37 & 37 & 38 & 41 & 39 & 41 & 42 & 43 & 44 & 44 & 47 & 52 & & 56 & 75 \\
Alekhine & 31 & 31 & 29 & 34 & 30 & 35 & 33 & 35 & 38 & 36 & 37 & 39 & 38 & 40 & 40 & 43 & 47 & 45 & 69 \\
Steinitz & 20 & 19 & 17 & 20 & 16 & 22 & 19 & 22 & 25 & 22 & 22 & 25 & 24 & 27 & 27 & 26 & 30 & 27 & 33 & \\
\hline
\end{tabular}

According to this predictor too, it is clear that the level of chess has been increasing through the years. A group of 4 players (Carlsen, Kramnik, Fischer, Kasparov) is ahead of the pack, while a group of 3 (Euwe, Alekhine and Steinitz) is trailing below (Steinitz being way below the others). The in-between players are close to each other. The results are not exactly the same as those found with the covariance indicator (Fischer for example has a better ranking here), however they are very close.

\subsubsection{Predicting the results of World Championship using the Markovian model}

Below, we compare the score predicted for World Championships by the Markovian predictor to the actual score and to the score predicted using ELO tables as we did for the accumulated conformance indicator in subsection 4.2.4 and for the covariance predictor in subsection 4.3.3. The results are also presented in Table 5 in column $M_{s}$.

The difference between the actual score and the Markovian predicted score is $3.6 \%$ on all Championships and of $4.4 \%$ on the 11 World Championships for which the ELO predictor is available. The Markovian predictor is thus the best of the three, even if the sample of available data is too small to have a definitive conclusion.

It would have been interesting to do a more thorough comparison of the three predictors, but this was out of reach of this study, as much more computing power is required.

\section{CONCLUSION}

Statistical studies give correlation information, they do not directly provide causality, and interpreting them requires some perspective. 
The cumulative conformance indicator presented in this article measures the capacity to play "like" a very strong computer program, even if its interpretation, as discussed in subsection 4.2.1, is a little bit more complex. Believing that this indicator is a measure of the real "strength" of a player is plausible, as I have demonstrated along this article that conformance was highly correlated with the game outcome, and we know that computer programs are currently much stronger than human beings. However, it is important to remember that the indicator has been built and verified only on world class players, and extending it to lesser players would require more experimentation. Moreover, we have seen that this indicator has some drawbacks, as it requires a delicate fitting to the data, and does not take into account the context of the moves played.

The distribution vector is in a sense "richer" than the scalar cumulative conformance indicator. However, it requires also to be fitted to the data, and its results regarding its ability to predict the results of matches seem less good than the other two.

The Markovian predictor gives very interesting prediction results which are better on this limited sample than the two other predictors and even better than the usual ELO predictor. It is the "purest" of the three, as it does not require any specific fitting, and the Markovian interpretation is apparently the "soundest" of the three. As it is an intrinsic predictor which does not depend on the evaluation of other players, it could be a possible replacement of the usual ELO predictor, even if a clear drawback is the fact that it is a composite predictor, which enables only to compare two players, but not to build simply a total order between players. However, it could be possible to build a ranking by simulating all possible confrontations between players of the same class, and averaging the results.

There remains a plethora of things to do. Below we mention seven of them.

- It is necessary to evaluate much more games, and this is definitely possible. A rough estimation done with Chessbase shows that there are certainly less than 500,000 regular time games which would have to be evaluated in order to assess all games where both players are above 2500 ELO. This is 25 times the number of games assessed in this study, but it is definitely within our grasp. On a Xeon E5-2680 v2 @ 2.80GHz processor, this would require around 500,000 hours of CPU time to have the quality of evaluation of this study which used old HE 6262 AMD processors. 500,000 hours is not much regarding the capacity of HPC centers: it would represent 40 hours for the CALMIP EOS computer, which was ranked 183 in the TOP 500 list as of 06/2014, and only 10 minutes on the Tianhe-2 (the speedup is completely linear with the number of cores as the problem is fully parallel). Even evaluating the complete Chessbase database after removing games with fast time controls is now possible.

- The database must be checked again. Properly filtering the database is a difficult problem. Finding time controls is usually difficult, and it is sometimes necessary to guess them from the name of the tournament. Also, cleaning up the history of the games to suppress move repetitions would probably be beneficial. Moreover there are little glitches (such as players who have exactly the same names, while they are not the same player) which should be solved before going further. Cooperating with people developing chess databases would definitely be a clear advantage.

- Results should be compared by using different chess engines to evaluate moves, to see what results are "engine dependent" or "engine independent". It is almost certain that some of them depend on the program used, as the evaluation function is different from program to program. However, what is probably more important is the similarity in the ranking of moves. Further experiments are required, even if there has already been such studies (Guid and Bratko, 2011), which mainly conclude that the ranking between chess programs is usually consistent. 
- More data should be gathered. It would have been beneficial to store more information during the search. This would have enabled to compute other indicators (such as Sullivan's complexity) and test other approaches, such as Haworth and Regan's.

- The conformance indicator, gain distribution vector or Markovian matrices should probably be computed separately for White and for Black, as players seem to play differently when they are playing as Black or as White. This is an aside, but interesting result, of this study.

- Some of the problems found in this study might be a consequence of the structure of the evaluation functions in chess, which cannot be mapped easily to the probability of winning a game. So, on the one hand, a parallel statistical work regarding evaluation functions could be performed in order to better understand this mapping. On the other hand, applying this methodology to a game such as Othello/Reversi with an engine using an evaluation function returning the probability of winning would also provide useful information.

- Results might depend on the fact that the model has been fitted ${ }^{36}$ to a particular type of players, namely world class players, and even more generally to human beings. The psychological biases that appear when playing as Black, or when playing a little recklessly in inferior positions would not appear in games between computers. So it would be extremely interesting to gather games played by computers at blitz level, and to see how the results are modified.

However, the intermediate results show that the level in chess has raised through the years, and that the young players of our days are extremely strong. This is probably to be expected: Magnus Carlsen was born in 1990, which means that he had at his disposal for training during almost all his life a small computing device at home which was stronger than any existing player ever, and databases containing all the games ever played. The drawback is probably that the current chess games are sometimes considered as "dull" by some commentators: there are very few mistakes made, and a single mistake is usually sufficient to lose the game. They probably look more and more like computer games, and the brilliance of play like the one of Misha Tal is probably now only an echo of the past. In comparison, the performance of players like Fischer are all the most impressive, as they are on par with this new generation, while they were far from having the same tools at their disposal.

It is also important to stress a last important point: the Markovian method presented here could be, in theory, used for any two-player game where an oracle (i.e., a computer program playing "much" better than human beings) is available. This currently covers a very large number of two-players games, as computer programs have become continuously stronger in the previous years, and there is no reason to believe that this trend is going to change. Thus it would be possible to use an identical "rating" system for all such games, that would have the same advantages (and drawbacks). Validating the Markovian model on other games such as reversi, checkers, or draughts is definitely something to do.

\section{Acknowledgments}

The author would like to thank the referees of this article, along with the former Editor of the ICGA Journal. Their comments were extremely informative, they spotted weaknesses in the paper that had to be addressed, experiments that had to be done, bibliographical references that had to be improved, and they greatly helped in improving this work.

\footnotetext{
${ }^{36}$ This is not true for the Markovian indicator, which is not fitted to the data.
} 


\section{REFERENCES}

Barnes, D., \& Castro-Hernandez, J. (2015). On the limits of engine analysis for cheating detection in chess. Computers and security, 48, 58-73.

Canbaz, S. (2015). Stockfish benchmarks. mycharhttps://sites.google.com/site/computerschess/ stockfish-chess-benchmarks.

CCRL website (2015). Computer chess rating list 40/40. http://www.computerchess.org.uk/ccrl/4040/.

Chessbase (2009). Breakthrough performance by pocket fritz 4 in Buenos Aires. http://en. chessbase.com/post/breakthrough-performance-by-pocket-fritz-4-in-Buenos-Aires.

Dailey, D., Hair, A., \& Watkins, M. (2013). Move similarity analysis in chess programs. Entertainment Computing, 5(3), 159-171. DOI:10.1016/j.entcom.2013.10.002

Elo, A. (1978). The rating of chess players past and present. Arco Publishing.

Fatta, G. D., Haworth, G., \& Regan, K. (2009). Skill rating by bayesian inference. Computational Intelligence \& Data Mining (CIDM), pp. 89-94, Institute of Electrical and Electronics Engineers. ISBN 9781424427659.

Ferreira, D. (2012). Determining the strength of chess players based on actual play. ICGA Journal, 35(1), 3-19.

Ferreira, D. R. (2013). The impact of the search depth on chess playing strength. ICGA Journal, 36(2), 67-80.

Glickman, M. (1995). A comprehensive guide to chess ratings. American Chess Journal, 3, 59-102.

Glickman, M., \& Jones, A. (1999). Rating the chess rating system. Chance, 12(2), 21-28.

Gomboc, D., Buro, M., \& Marsland, T. A. (2005). Tuning evaluation functions by maximizing concordance. Theor Comput Sci, 349(2), 202-229. ISSN 0304-3975. http://dx.doi.org/10.1016/ j.tcs.2005.09.047.

Guid, M. (2010). Search and knowledge for human and machine problem solving. Ph.D. thesis, University of Ljubljana, Slovenia.

Guid, M., \& Bratko, I. (2006). Computer analysis of world chess champions. ICGA Journal, 29(2), 3-14.

Guid, M., \& Bratko, I. (2007). Factors affecting diminishing returns for searching deeper. ICGA Journal, 30(2), 65-73.

Guid, M., \& Bratko, I. (2008). How trustworthy is crafty analysis of world chess champions? ICGA Journal, 31(3), 131-144.

Guid, M., \& Bratko, I. (2011). Using heuristic-search based engines for estimating human skill at chess. ICGA Journal, 34(11), 71-81.

Harkness, K. (1967). Official chess handbook. McKay.

Haworth, G., Regan, K., \& Fatta, G. D. (2010). Advances in computer games, vol. 6048 of lecture notes in computer science, Chapter Performance and Prediction: Bayesian Modelling of Faillible Choice in Chess, pp. 99-110. Springer. 
Heinz, E. A. (2001a). Follow-up on self play, Deep search and diminishing returns. ICGA Journal, 26(2), 75-80.

Heinz, E. A. (2001b). Self play, deep search and diminishing returns. ICGA Journal, 24(2), 75-79.

Hyatt, R. (1997). Crafty goes deep. ICCA Journal, 20(2), 79-86.

Keene, R., \& Divinsky, N. (1989). Warriors of the mind: A quest for the supreme genius of the chess board. Batsford Limited. ISBN-13:978-0951375709.

Levene, M., \& Bar-Ilan, J. (2005). Comparing move choices of chess search engines. ICGA Journal, 28(2), 67-76.

Levy, D. (2005). 8:4 final score for the machines - What next? http://dx.doi.org/10.1016/j.tcs.2005. 09.047 .

Nelder, J., \& Mead, R. (1965). A simplex method for function minimization. Computer Journal, 7(4), 308-313.

Riis, S. (2006). Review of "Computer Analysis of World Champions". http://en.chessbase.com/ post/computer-analysis-of-world-champions.

Snir, M., Otto, S., Huss-Lederman, S., Walker, D., \& Dongarra, J. (1995). MPI, the complete reference. MIT Press. ISBN 0-262-69215-5.

Sonas, J. (2005). Chessmetrics website. http://www.chessmetrics.com.

Sullivan, C. (2008). True Chess Compares the Champions. http://www.chessmetrics.com.

Swedish Chess Computer Association (2015). Swedish Chess Program Rating List. http://ssdf. bosjo.net/. 